\title{
Immune-related gene expression in skin, inflamed and keloid tissue from patients with keloids
}

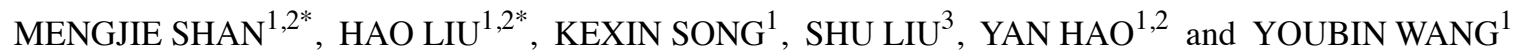 \\ ${ }^{1}$ Department of Plastic Surgery, Peking Union Medical College Hospital; \\ ${ }^{2}$ Graduate School, Chinese Academy of Medical Sciences and Peking Union Medical College, Beijing 100730; \\ ${ }^{3}$ Department of General Surgery, Civil Aviation General Hospital, Beijing 100123, P.R. China
}

Received July 16, 2021; Accepted November 1, 2021

DOI: $10.3892 / \mathrm{ol} .2022 .13192$

\begin{abstract}
Keloids are a tumor-like fibroproliferative skin disease that could cause disfigurement and disability. The pathological mechanisms underlying this condition remain unclear, particularly the progression from normal healthy skin to inflammatory skin tissue, then keloid. In the present study, three immune-related gene expression profiling datasets, were obtained from normal skin tissue ( $\mathrm{N}$ group), inflamed tissue (I group) and keloid tissue samples from patients with keloids (K group). This sample grouping represents the primary steps of keloid formation, from normal to inflammatory, and finally to keloid tissue. The expression levels of immune-related genes were analyzed, and the differentially expressed genes (DEGs) between the three groups were compared. Protein-protein interaction networks were established using Cytoscape. Gene Ontology (GO) and Kyoto Encyclopedia of Genes and Genomes pathway enrichment analyses were carried out to determine the main functions associated with the DEGs and keloidassociated pathways. The results identified hub genes in the $\mathrm{N}$ and I groups, including $\mathrm{C}-\mathrm{C}$ motif chemokine receptor (CCR) 1, CCR7, CD40 ligand, C-X-C motif chemokine ligand 9, IL-6 and IL-10. The hub genes in the I and the $\mathrm{K}$ groups included IL-10, IL-6, IL-13 and CD86. The expression levels of these genes were verified using reverse transcription-quantitative PCR. The results demonstrated that IL-6 expression levels were significantly increased in the I group compared with
\end{abstract}

Correspondence to: Professor Youbin Wang, Department of Plastic Surgery, Peking Union Medical College Hospital, 1 Shuaifuyuan Road, Beijing 100730, P.R. China

E-mail: wybenz@sina.com

*Contributed equally

Abbreviations: DEG, differentially expressed gene; GO, Gene Ontology; KEGG, Kyoto Encyclopedia of Genes and Genomes; $\mathrm{H} \& \mathrm{E}$, hematoxylin and eosin; PPI, protein-protein interaction; BP, biological process; $\mathrm{CC}$, cell component; $\mathrm{MF}$, molecular function

Key words: keloid, immune-related gene, differentially expressed genes, tumor epigenetics, $\mathrm{C}-\mathrm{C}$ motif chemokine receptor 7 the $\mathrm{N}$ group $(\mathrm{P}=0.0111)$. CCR7 levels significantly differed between all three groups $(\mathrm{P}<0.017)$. The results of $\mathrm{GO}$ analysis suggested that the hub genes in the I and $\mathrm{N}$ groups may be associated with 'regulation of lymphocyte activation' and 'T-cell activation'. Similar results were also observed between the I and K groups, which may play an important role in keloid initiation and formation. In conclusion, CCR7, IL-10 and IL-6 may be important in keloid initiation and formation. These findings provided insight into the pathogenesis of keloids and may help identify novel immune-related therapeutic targets for this condition.

\section{Introduction}

Keloids are abnormal fibrous hyperplasias that exceed the initial injury area and invade adjacent healthy skin (1-3). Due to limitations in the understanding of the pathogenesis of keloids, clinical research progress into the prevention and treatment of keloids has been relatively slow. All current treatments are associated with recurrence. Therefore, it is necessary to further examine keloid pathogenesis and lay the foundation for the clinical exploration of new treatment options. The study of disease-causing genes is indispensable in this regard. The genetic theory of keloids has mainly been founded on the discovery that keloids run in families $(1,2)$. Keloids are highly prevalent among certain ethnic groups $(1,2)$. However, numerous patients with keloids have no family history of this condition $(3,4)$. Thus, the characteristics of genes associated with keloid formation require further exploration.

Keloid scars may result from skin lesions and irritants, including trauma, insect bites, burns, surgery, skin punctures, acne, hair folliculitis and herpes zoster infection (5). Infection is also an important pathogenic factor in keloid formation (5). Keloids often arise from skin infections, such as acne. It has also been revealed that the number of inflammatory cells and fibroblasts in the reticular layer of keloids is elevated (5). In addition, pro-inflammatory cytokines, such as IL-1, IL-6 and TNF- $\alpha$, are upregulated in keloid tissue, suggesting that pro-inflammatory gene expression is increased and localized inflammation is present in patients with keloids (6). The involvement of other pro-inflammatory cytokines, such as IL-4, IL-10 and IL-13, has also been reported in the literature $(7,8)$. However, the association between the expression of 
various inflammatory factors and local gene expression is not well understood.

In the present study, an analysis of immune-related genes, the expression profiles and differentially expressed genes (DEGs) found at different stages of keloid development was used to identify hub genes. To describe the tumor gene expression characteristics of keloid development, healthy skin tissue, inflamed tissue and keloid tissue samples were collected from patients with keloids. A bioinformatics approach was adopted to explore the differences in the expression of immune-related genes in these samples and to identify potential hub genes at different stages of keloid development.

\section{Materials and methods}

Patients. The present study was approved (approval no.JS-2907) by The Medical Ethics Committee of Peking Union Medical College Hospital in Beijing, China. Written and photographic informed consents were obtained from all participants. A total of 9 patients with keloids and inflammation next to their keloid mass and healthy tissue around the keloid mass were enrolled between January 2019 and March 2020. Basic demographic information (sex and age) of the participants was collected, and the condition of their keloids was assessed using the modified Vancouver scar scale (9) according to the type of keloid lesions they had (Tables I and II). Patients $<18$ or $>60$ years of age were excluded. Patients with severe systemic disease were also excluded. No patients had other systemic disorders, or received any drugs or other treatments that may affect the study results (such as corticosteroids, 5-fluorouracil injection or radiotherapy). There were 4 male and 5 female patients, and their ages ranged from 24-37 years. The patients were divided into two groups. Group 1 included 5 patients whose tissue samples were used for high-throughput sequencing (Table I). Group 2 included 4 patients whose tissue samples were used for experimental verification (Table II). All samples were collected from the chest region. Keloid samples (K group; K7, $\mathrm{K} 8, \mathrm{~K} 9, \mathrm{~K} 14, \mathrm{~K} 36$ ) were obtained from the inner zone of the keloid tissue removed after surgery. Healthy skin samples ( $\mathrm{N}$ group; N7, N8, N9, N14, N36) were obtained from healthy skin tissue that had to be removed during keloid resection. Inflamed tissue samples (I group; I7, I8, I9, I14, I36) were obtained from the inflamed skin tissue after acute-stage in patients with keloids and inflammatory lesions (Fig. S1) occurring outside the keloid mass, which were removed during keloid resection. The distance between the keloid and the surrounding healthy skin tissue was 2-3 $\mathrm{mm}$.

Hematoxylin and eosin $(H \& E)$ staining. $H \& E$ staining was performed on the tissue samples. Following $10 \%$ formaldehyde (Thermo Fisher Scientific, Inc.) fixation $\left(12 \mathrm{~h}, 25^{\circ} \mathrm{C}\right)$, alcohol dehydration, xylene transparency and paraffin embedding, the tissue was cut into thin slices (5-8 $\mu \mathrm{m}$ in thickness). The slices were then blanched in hot water, fixed to slides and dried at $45^{\circ} \mathrm{C}$ in an incubator. Before staining, the paraffin wax was gradually removed using xylene. Following another dehydration process, the slides were stained $\left(3 \mathrm{~min}, 25^{\circ} \mathrm{C}\right)$ with $\mathrm{H} \& \mathrm{E}$.

Expression levels of immune-associated genes. The samples from Group 1 were used for high-throughput sequencing. The
Oncomine Immune Response Research Assay kit (10) (Thermo Fisher Scientific, Inc.; cat. no. A32881), was used according to the manufacturer's protocol. TaqMan ${ }^{\circledR}$ Quantitation kit (Thermo Fisher Scientific, Inc.; cat. No. 4468802) was used for quantification and dilution of the sample to $100 \mathrm{pM}$. Agarose gel electrophoresis was used to identify RNA integrity. Ion $520^{\mathrm{TM}}$ \& Ion $530^{\mathrm{TM}}$ kit (Thermo Fisher Scientific, Inc.; cat. no. A27751) was used for sequencing. The nucleotide length was 100-200 bp and the sequencing direction was non-specific. The data were analyzed by $\mathrm{R}$ package (Version 3.6.1, rstudio. com/products/rstudio/download/) (11). incipal component analysis (PCA). The analysis of DEG profiles using $\mathrm{R}$ package (version 3.4.3, R Foundation for Statistical Computing) and then dimension reduction analysis was performed. PCA is one of the most widely used data dimension reduction algorithms. PCA maps $\mathrm{N}$-dimensional features to K-dimensional features, which are new orthogonal features, also known as principal components, and are K-dimensional features reconstructed on the basis of the original N-dimensional features. The aim of PCA is to identify a set of mutually orthogonal coordinate axes sequentially from the original space. The selection of new coordinate axes is closely related to the data itself. This is equivalent to retaining only the dimension features containing most of the variance, while ignoring the feature dimensions containing almost 0 variance, so as to realize the dimensionality reduction processing of data features. The DEGs based on the I, K and $\mathrm{N}$ groups were analyzed.

Identification and functional annotation of DEGs. DEGs were introduced into Kyoto Encyclopedia of Genes and Genomes (KEGG) (12) and enriched into the signaling pathway map. Significant DEGs in the three datasets were defined as genes with $\mathrm{P}<0.05$ and $\log _{2}$ (fold change) $>1.5$ or $<1.5$. The $\mathrm{R}$ package was used to create the volcano map. The Database for Annotation, Visualization and Integrated Discovery (DAVID) (13) was used for functional and pathway enrichment analysis of DEGs. KEGG is a database used to help understand complex biological processes.

Determination of the hub genes. 'Multiple proteins' option was selected to input proteins. Then the 'SEARCH' option was selected to obtain the PPI map. STRING (14) is a database that can predict protein-protein interactions (PPIs). It has powerful protein performance retrieval functions and is usually used for proteomics research, research into the molecular mechanisms of disease and the discovery of new drug targets. Cytoscape software (version 3.5.1) was initially used to establish a PPI network (15). MCODE (16) (version 3.5.1) and Cytohubba (version 3.5.1) was then used to further identify modules $(17,18)$. A hub gene is a gene that plays a crucial role in biological processes. In related pathways, the regulation of other genes is often affected by this gene (19). Therefore, a hub gene is often an important target and research hotspot. Subsequently, the hub genes were screened according to the network topology.

Reverse transcription-quantitative PCR (RT-qPCR). Tissue samples from the I, $\mathrm{K}$ and $\mathrm{N}$ groups ( $\mathrm{n}=4$ in each group) were used for RT-qPCR validation in three independent experimental repeats. The primers used in this study are revealed in Table III. Each tissue sample $(200 \mathrm{mg})$ was placed into 
Table I. Characteristics of patients of group 1.

\begin{tabular}{lclcc}
\hline Patients $^{\mathrm{a}}$ & $\begin{array}{c}\text { Age of onset } \\
\text { (years) }\end{array}$ & Sex & mVSS & $\begin{array}{c}\text { Onset time } \\
\text { (years) }\end{array}$ \\
\hline K7, N7, I7 & 24 & Female & 11 & 11 \\
K8, N8, I8 & 32 & Male & 10 & 8 \\
K9, N9, I9 & 37 & Female & 10 & 16 \\
K14, N14, I14 & 21 & Male & 9 & 6 \\
K36, N36, I36 & 30 & Female & 10 & 9 \\
\hline
\end{tabular}

${ }^{\mathrm{a}} \mathrm{K}, \mathrm{N}$ and I samples were collected from a total of 5 patients. mVSS: The Modified Vancouver Scar Scale is used for the descriptive assessment of keloids, including melanin $(\mathrm{M})$, height $(\mathrm{H})$, vascularity $(\mathrm{V})$ and pliability $(\mathrm{P})$.

Table II. Characteristic of patients of group 2.

\begin{tabular}{lclcc}
\hline Patients $^{\mathrm{a}}$ & $\begin{array}{c}\text { Age of onset } \\
\text { (years) }\end{array}$ & Sex & mVSS & $\begin{array}{c}\text { Onset time } \\
\text { (years) }\end{array}$ \\
\hline K1, N1, I1 & 25 & Female & 8 & 7 \\
K2, N2, I2 & 37 & Male & 10 & 9 \\
K3, N3, I3 & 26 & Female & 9 & 13 \\
K4, N4, I4 & 33 & Male & 11 & 10 \\
\hline
\end{tabular}

${ }^{\mathrm{a}} \mathrm{K}, \mathrm{N}$ and I samples were collected from a total of 4 patients. mVSS: The Modified Vancouver Scar Scale is used for the descriptive assessment of keloids, including melanin $(\mathrm{M})$, height $(\mathrm{H})$, vascularity (V) and pliability (P).

a 1.5-ml Eppendorf tube, and $1 \mathrm{ml} \mathrm{TRIzol}{ }^{\circledR}$ (Invitrogen; Thermo Fisher Scientific, Inc.) was added to the tube. After blending vigorously for $30 \mathrm{sec}, 0.2 \mathrm{ml}$ chloroform was added, and the tube was shaken vigorously for $30 \mathrm{sec}$. The samples were incubated at room temperature for $3 \mathrm{~min}$, then centrifuged at $12,000 \mathrm{x}$ g at $4^{\circ} \mathrm{C}$ for $15 \mathrm{~min}$. In total, $\sim 0.5 \mathrm{ml}$ of the colorless, upper aqueous phase was transferred into a fresh Eppendorf tube. An equal volume of isopropanol was added, and the samples were incubated at $-20^{\circ} \mathrm{C}$ for $30 \mathrm{~min}$. The samples were then centrifuged at $12,000 \times \mathrm{g}$ at $4^{\circ} \mathrm{C}$ for $10 \mathrm{~min}$. Traces of precipitated RNA could be observed at the bottom of the tube. A volume of $1 \mathrm{ml} \mathrm{75 \%} \mathrm{ethanol} \mathrm{was} \mathrm{added,}$ and the tubes were shaken. The samples were then centrifuged at 7,500 x g at $4^{\circ} \mathrm{C}$ for $10 \mathrm{~min}$. The supernatant was discarded. The residual liquid was carefully absorbed using filter paper, and the tube was then dried at room temperature for 5-10 min. The precipitate was dissolved in $20 \mu \mathrm{l}$ DEPC-treated water (Thermo Fisher Scientific, Inc.). The concentration and purity of RNA were then measured, and each sample was then stored at $-70^{\circ} \mathrm{C}$. The following steps were completed according to the instructions of the HiScript ${ }^{\circledR}$ II One Step RT-PCR kit (Vazyme Biotech Co., Ltd.): $50^{\circ} \mathrm{C}$ for $30 \mathrm{~min} ; 94^{\circ} \mathrm{C}$ for $3 \mathrm{~min} ; 94^{\circ} \mathrm{C}$ for $10 \mathrm{sec}, 55^{\circ} \mathrm{C}$ for $1 \mathrm{~min}, 35$ cycles; $72^{\circ} \mathrm{C}$ for $5 \mathrm{~min}, 4^{\circ} \mathrm{C}$ hold. The fluorophore was SYBR Green kit (Vazyme Biotech Co., Ltd.). The expression levels of the target genes were then obtained using $2^{-\Delta \Delta \mathrm{Cq}}$ method (20).
Table III. Primers and their sequences for PCR analysis.

\begin{tabular}{ll}
\hline Primer & \multicolumn{1}{c}{ Sequence (5'-3') } \\
\hline GAPDH-F & GGAAGCTTGTCATCAATGGAAATC \\
GAPDH-R & TGATGACCCTTTTGGCTCCC \\
CCR1-F & GACTATGACACGACCACAGAGT \\
CCR1-R & CCAACCAGGCCAATGACAAATA \\
CCR7-F & TGAGGTCACGGACGATTACAT \\
CCR7-R & GTAGGCCCACGAAACAAATGAT \\
CD40LG-F & ACATACAACCAAACTTCTCCCCG \\
CD40LG-R & GCAAAAAGTGCTGACCCAATCA \\
CD86-F & CTGCTCATCTATACACGGTTACC \\
CD86-R & GGAAACGTCGTACAGTTCTGTG \\
CXCL9-F & CCAGTAGTGAGAAAGGGTCGC \\
CXCL9-R & AGGGCTTGGGGCAAATTGTT \\
IL-6-F & GCAATAACCACCCCTGACCCA \\
IL-6-R & CAGAAGAAGGAATGCCCATTAACAA \\
IL-10-F & GACTTTAAGGGTTACCTGGGTTG \\
IL-10-R & TCACATGCGCCTTGATGTCTG \\
MMP2-F & TACAGGATCATTGGCTACACACC \\
MMP2-R & GGTCACATCGCTCCAGACT \\
IL-13-F & CCTCATGGCGCTTTTGTTGAC \\
IL-13-R & TCTGGTTCTGGGTGATGTTGA \\
\hline
\end{tabular}

F, forward; R, reverse.

Western blot analysis. Tissue samples from the patients in group 2 were then extracted. The tissue blocks were washed 2-3 times with pre-cooled PBS, and lysis buffer (Beijing Solarbio Science \& Technology Co., Ltd.) was added to isolate the total protein. Protein samples (50-100 $\mu \mathrm{g} /$ lane) were resolved using SDS-PAGE on $10 \%$ gels, and then transferred to PVDF membranes. Subsequently, the membranes were blocked with $5 \%$ skimmed milk for $1 \mathrm{~h}$ (room temperature). The primary antibody [anti-C-C motif chemokine receptor 7 (CCR7) polyclonal antibody; 1:600; cat. no. 25898-1-AP; or anti-GAPDH (1:20,000; cat. no. 10494-1-AP)] was applied dropwise, and the samples were incubated overnight at $4^{\circ} \mathrm{C}$. HRP-conjugated Affinipure Goat Anti-Rabbit IgG ( $\mathrm{H}+$ L) secondary antibody (1:2,500; cat. no. SA00001-2) was then added for $2 \mathrm{~h}$ at room temperature. All the antibodies were from ProteinTech Group, Inc. GAPDH was used as the internal reference protein. ImageJ software (National Institutes of Health, version 1.8.0) was used to analyze the gray values of the protein bands, which were normalized to GAPDH.

Statistical analysis. The statistical analysis was carried out using SPSS software version 22.0 (IBM Corp.). The unpaired t-test was used to compare the differences between two groups. One-way ANOVA was used to compare the differences between three groups. Following ANOVA, Bonferroni's correction was used to reduce the uncertainty of the results obtained. P-values were considered to be statistically significant at a Bonferroni corrected $\mathrm{P}<0.017$. $\mathrm{P}<0.017$ was considered to indicate a statistically significant difference. 


\section{Results}

Histology of healthy, inflamed and keloid tissue from patients with keloids. H\&E staining of healthy, inflamed and keloid tissue was analyzed to determine the tissue characteristics and any morphological differences between the groups (Fig. 1). The three groups of samples were not consistent in morphology, representing the morphological changes of the disease from normal skin to inflammatory tissue, and finally keloid formation. The epidermis of healthy skin tissue was thinner, while the dermis of healthy skin tissue around the keloid mass was relatively loose, with irregular collagenous bundles and fewer cells. The skin capillaries were scattered throughout the fibrous tissue (Fig. 1A). In the inflamed tissue, the epidermal layer was thicker, and the number of inflammatory cells increased considerably. The collagen in the dermis was disordered and dense. Scattered blood vessels were visible in the fibrous tissue and appeared dilated and congested (Fig. 1B). In keloid tissue, there were clear abnormalities and heterogeneity in the epidermal layer. The peripheral epidermal layer was subdivided, branch-like and visibly penetrated into the dermis, forming a deep canine tooth-like appearance, while the central epidermal layer was thick and flat. Collagen fibers in the superficial layer of keloids were small and parallel to the epidermis. The deep layer of the keloid was characterized by a dense extracellular matrix and disordered collagen fibers. Microvascular congestion and dilatation were not visible, although the number of blood vessels was greater than that in healthy tissue. There were also fewer inflammatory cells than in inflammatory tissue (Fig. 1C).

Data quality evaluation. PCA was used to verify the repeatability of the data within the groups. This analysis demonstrated that the data in groups I and $\mathrm{N}$ were repeatable (Fig. 2A). PCA also suggested that the distance between the samples in the same group was very small and the that distance between samples in different groups was relatively large in the PC1 dimension (Fig. 2B). There were partial data intersections between the $\mathrm{N}$ and the $\mathrm{K}$ groups (Fig. 2C). Through dimension reduction analysis, the three groups of data were grouped clearly, and further gene expression analysis was carried out.

Identification of DEGs among the three groups. To identify differences in tumor gene expression between the N, I and $\mathrm{K}$ groups, volcano maps were created (Fig. 3). There were 74 upregulated DEGs in the I group compared with the $\mathrm{N}$ group, and 20 downregulated (Fig. 3A). In addition, 45 DEGs were upregulated in the I group compared with the $\mathrm{K}$ group, whereas 37 DEGs were downregulated (Fig. 3B). There were 20 upregulated and 14 downregulated DEGs in the $\mathrm{N}$ group compared with the $\mathrm{K}$ group (Fig. 3C). Identification of DEGs among the three groups was used to further identify hub genes. There were several different hub genes in group I compared with group $\mathrm{K}$ and group $\mathrm{N}$, which may also indicate that tissue inflammation is an important pathological stage in keloid formation.

Analysis of the PPI network and hub genes. A PPI network of the DEGs was constructed (Fig. 4A-C), and the most significant modules (Fig. S2) and networks of hub genes (Fig. 4D-F) were
A

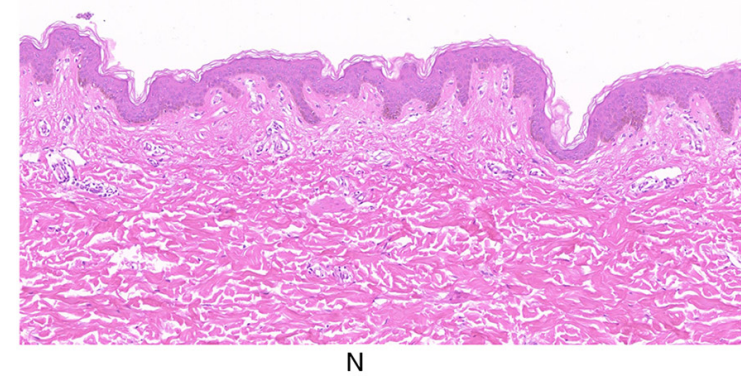

B

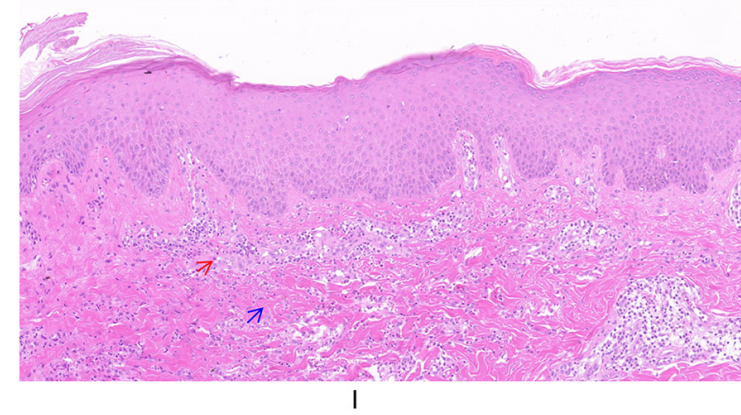

C

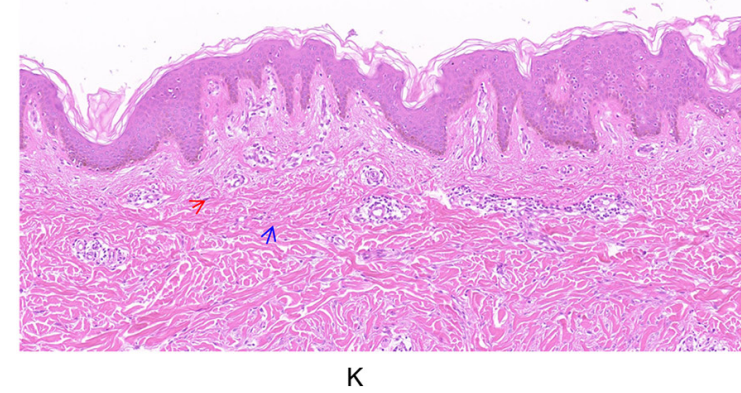

Figure 1. Histological images of healthy skin, inflamed tissue and keloid tissue from the same patient. The three groups of samples were not consistent in morphology, representing the morphological changes of the disease from normal skin to inflammatory tissue, and finally forming keloids (A) H\&E staining of group N. Magnification, x100. (B) H\&E staining of group I. Magnification, x100. (C) H\&E staining of group K. Magnification, x100. The deep purple cells indicated by the red arrow are inflammatory cells. The blue arrows indicate collagen fibers. H\&E, hematoxylin and eosin.

identified using Cytoscape software. MCODE and Cytohubba are two algorithms that screen for potential hub genes. The most commonly used algorithm is Cytohubba, and thus our subsequent validation was based on this. A total of 10 genes were identified as hub genes with a degree of $\geq 10$. In order to provide more original data, the promising hub genes obtained by bioinformatics algorithm analysis listed in Table IV require further PCR validation. The hub genes in the I and the $\mathrm{N}$ group included CCR1, CCR2, SELL, IL10, CCR7, CD40LG, CD69, CXCL8, IL-6 and CXCL9 (Fig. 4D; Table IV). All 10 hub genes were upregulated. The hub genes in the I and the $\mathrm{K}$ group included IL-10, ITGAM, ITGAX, IL-2, IL-4, IL-6, IL-13, IL-17A, FOXP3 and CD86 (Fig. 4E; Table V). Of these, three were downregulated, including ITGAM, ITGAX and FOXP3. The other seven genes were upregulated. Hub genes in the $\mathrm{N}$ and the $\mathrm{K}$ group included CD276, IL-6, S100A8, FCGR2B, TWIST1, CD68, TYROBP, MMP2, SNAI2 and TGFB1 (Fig. 4F; Table VI). These 10 hub genes were 
A

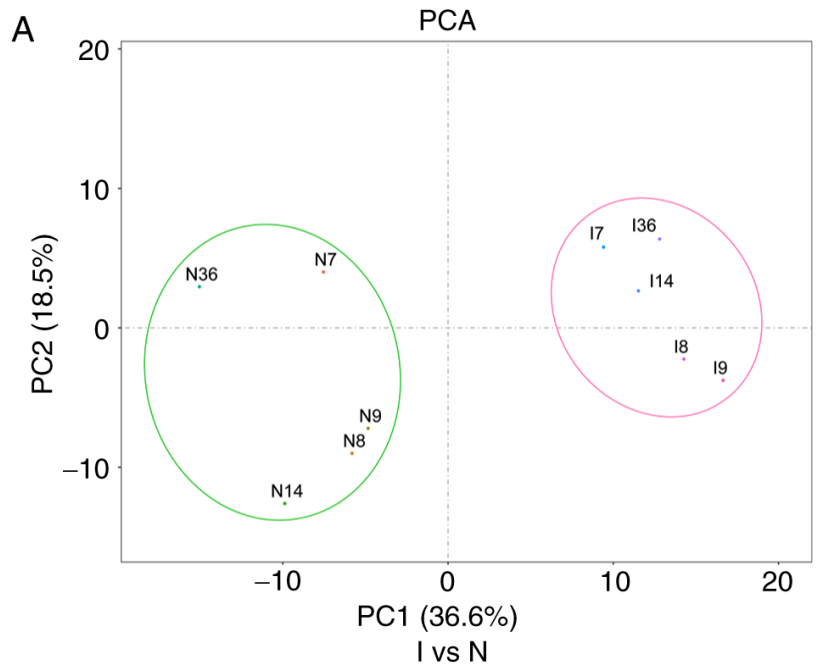

B

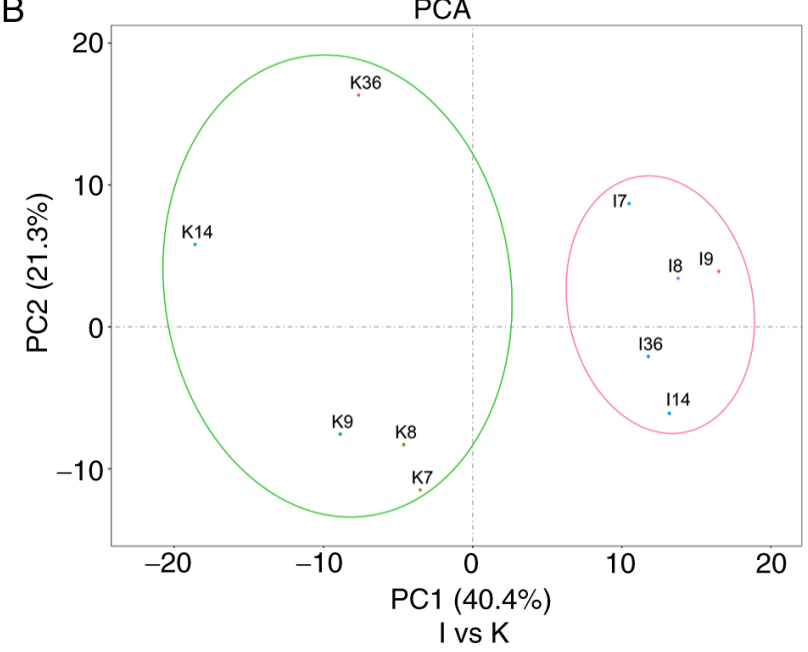

C

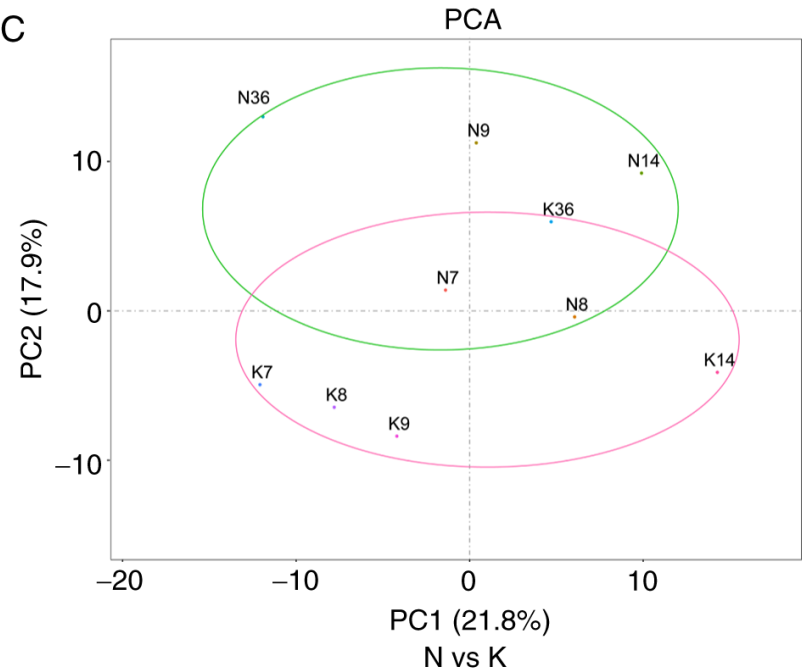

Figure 2. PCA is one of the most widely used data dimension reduction algorithms. By calculating the covariance matrix of the data matrix, the eigenvalue eigenvector of the covariance matrix is obtained, and the matrix composed of the corresponding eigenvectors of $k$ features with the largest eigenvalue (i.e., the largest variance) is selected. In this way, the data matrix can be transformed into the new space and the dimension reduction of data features can be realized. (A) PCA of samples between groups I and N. In the figure, principal component 1 (PC1) and principal component 2 (PC2) are used as the $\mathrm{X}$-axis and $\mathrm{Y}$-axis, respectively, to draw the scatter diagram, where each point represents a sample. In such a PCA diagram, the farther the two samples are from each other, the greater the difference is between the two samples in terms of gene expression patterns. (B) PCA of groups I and K. (C) PCA of groups N and K. PCA, principal component analysis.

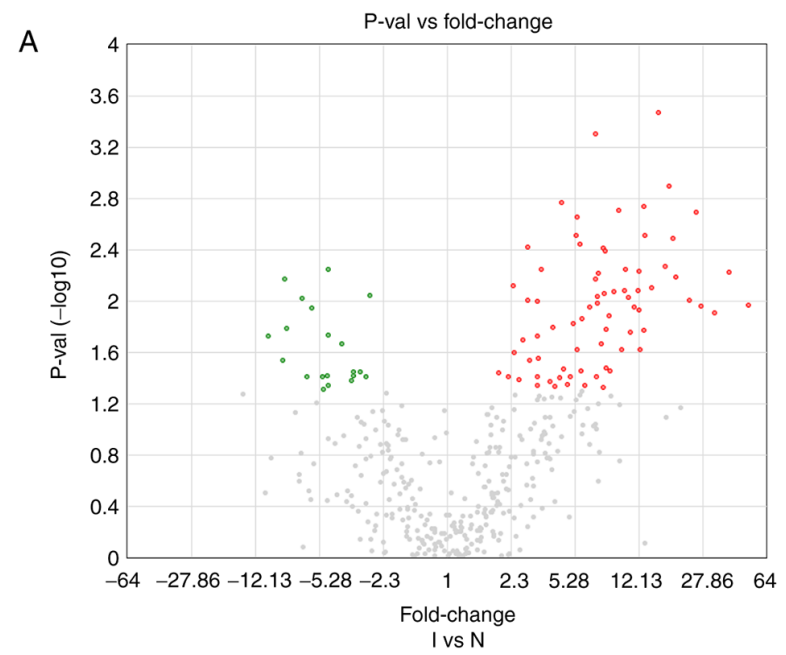

B
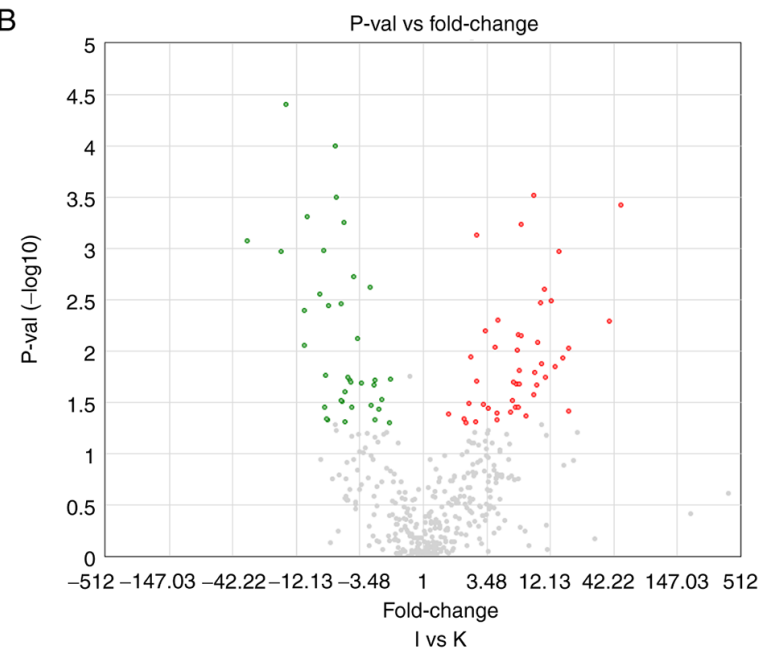

C

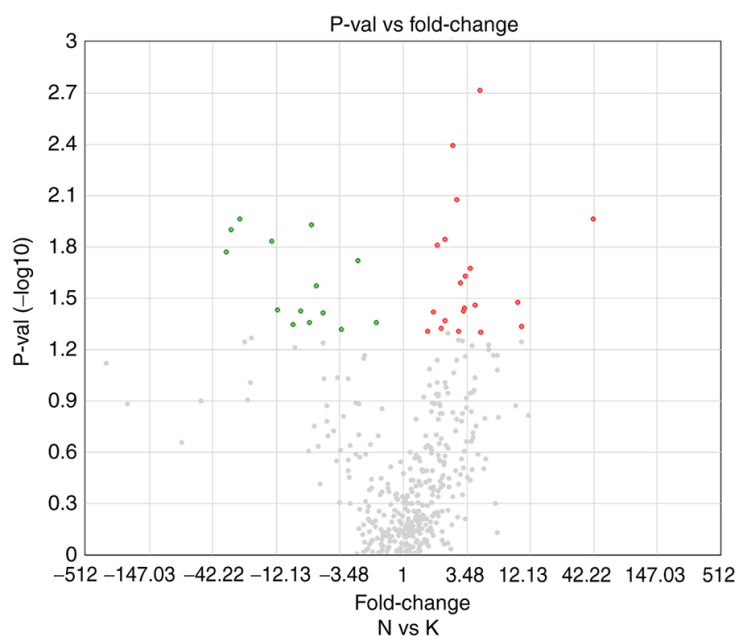

Figure 3. (A) A volcano map between groups I and N. The horizontal and vertical coordinates represent the average value of each different gene expression between groups I and $\mathrm{N}$. The cutoff for $\log 2$ fold change $>1.5$ or $<-1.5$ and $\mathrm{P}<0.05$ were the screening criteria. Significantly upregulated DEGs are shown in red, and significantly downregulated DEGs are shown in green. (B) Volcano map plot between groups I and K. (C) Volcano map between groups $\mathrm{N}$ and $\mathrm{K}$.

upregulated. MCODE detected densely connected regions of large PPI networks that may represent molecular complexes (Fig. S1). These hub genes predicted using bioinformatics may be important in keloid formation. 
A

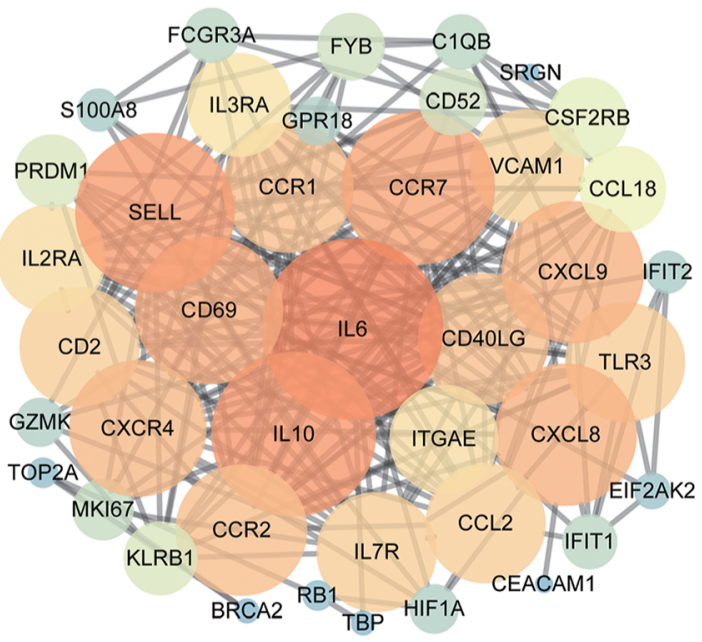

I vs $\mathrm{N}$

C

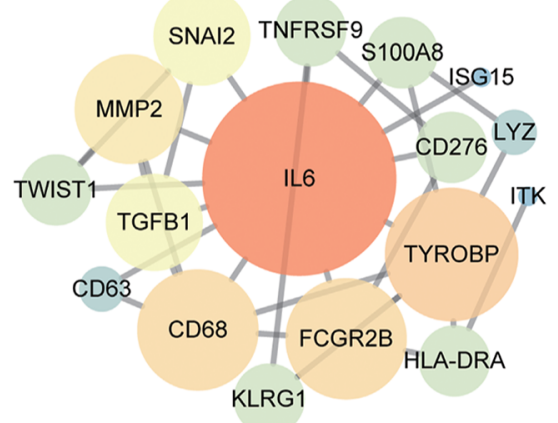

$\mathrm{N}$ vs K

E

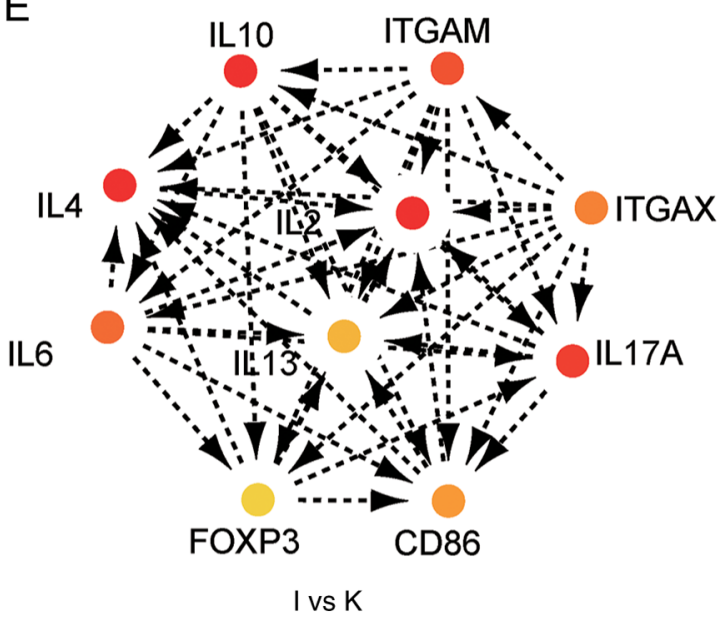

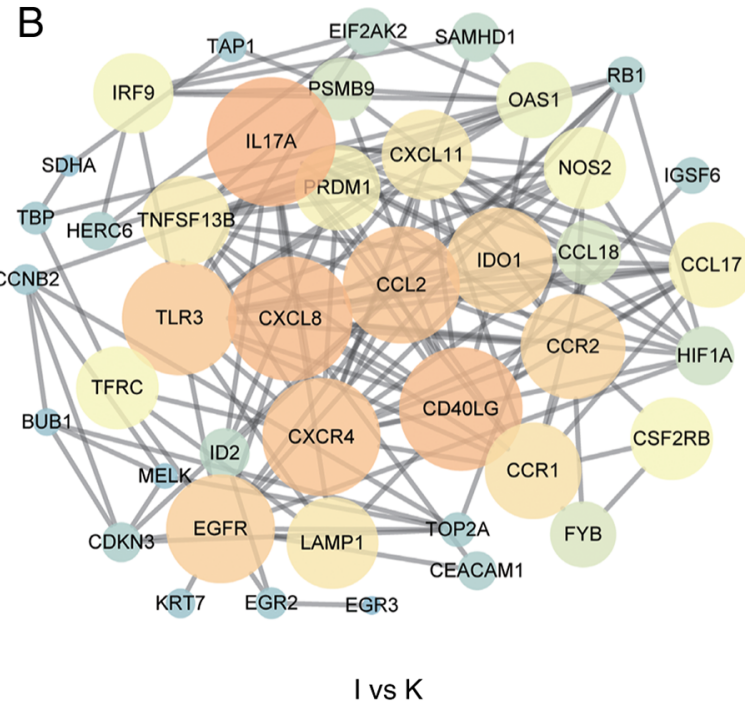

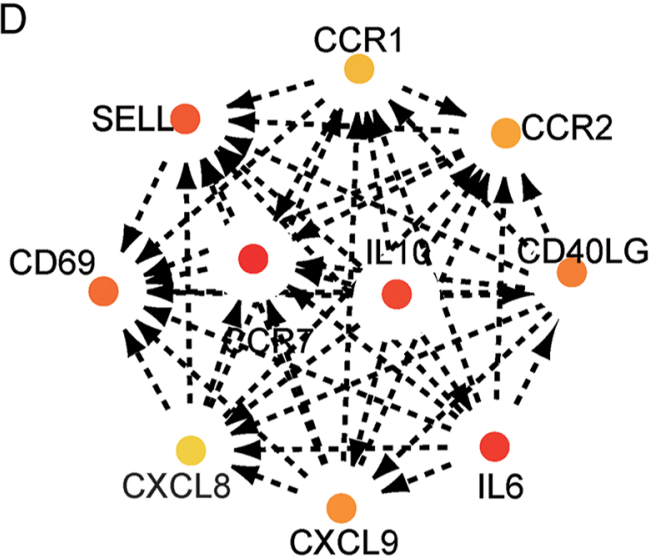

I vs $\mathrm{N}$

F

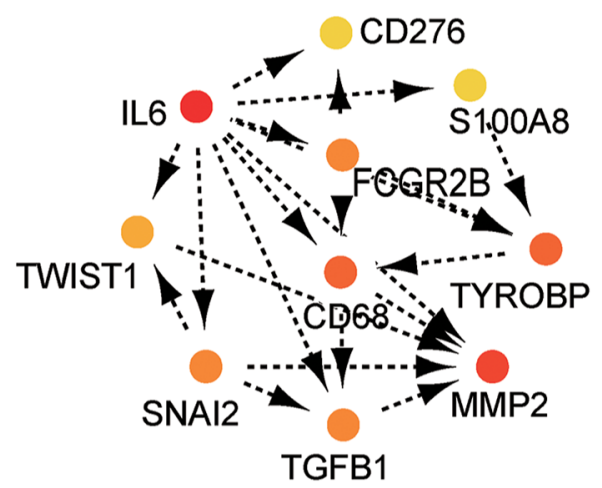

$\mathrm{N}$ vs K

Figure 4. PPI constructs the interactions between known and predicted proteins, and calculates the co-expression relationships of genes and function interaction network among genes. (A) PPI network of DEGs in groups I and N. (B) PPI network of DEGs in groups I and K. (C) PPI network of DEGs in groups N and K. (D) The hub genes were identified from the PPI network in groups I and N. (E) The hub genes were identified from the PPI network in groups I and K. (F) The hub genes were identified from the PPI network in groups $\mathrm{N}$ and $\mathrm{K}$. The larger circle, the darker color, the more it is associated with surrounding molecules. The more important the gene, the easier it is to screen out. DEG, differentially expressed gene; PPI, protein-protein interaction.

Functional annotation of DEGs using Gene Ontology $(G O)$ and KEGG analyses. Cutoff values of $\log _{2}$ fold change $>1.5$ or $<-1.5$ and $\mathrm{P}<0.05$ were used as the screening criteria for GO and KEGG analyses. GO analysis revealed that, compared with the $\mathrm{N}$ group, the I group was enriched in DEGs associated with biological process terms (BPs), 
Table IV. Differentially expressed genes between group I and group $\mathrm{N}$.

\begin{tabular}{lccc}
\hline Gene symbol & Fold change & P-value & FDR P-value \\
\hline CCR1 & 6.91 & 0.0005 & 0.0992 \\
SELL & 27.07 & 0.0109 & 0.1023 \\
CCR2 & 10.06 & 0.0083 & 0.1023 \\
CD69 & 50.44 & 0.0108 & 0.1023 \\
CCR7 & 8.33 & 0.0352 & 0.1858 \\
IL10 & 6.34 & 0.011 & 0.1023 \\
CD40LG & 25.48 & 0.002 & 0.1023 \\
CXCL8 & 15.68 & 0.0003 & 0.0992 \\
IL6 & 18.85 & 0.0032 & 0.1023 \\
CXCL9 & 9.34 & 0.0019 & 0.1023 \\
\hline
\end{tabular}

FDR, false discovery rate.

Table V. Differentially expressed genes between group I and group K.

\begin{tabular}{lccc}
\hline Gene symbol & Fold change & P-value & FDR P-value \\
\hline IL10 & 3.5 & 0.0632 & 0.2704 \\
ITGAM & -1.95 & 0.4047 & 0.6442 \\
IL4 & 1.03 & 0.4634 & 0.6933 \\
IL2 & 3.42 & 0.3898 & 0.6351 \\
ITGAX & -1.43 & 0.2395 & 0.5070 \\
IL6 & 4.15 & 0.1936 & 0.4479 \\
IL13 & 1.4 & 0.6538 & 0.8268 \\
IL17A & 3.55 & 0.0361 & 0.2111 \\
FOXP3 & -5.51 & 0.0598 & 0.2704 \\
CD86 & 4.52 & 0.0914 & 0.3307 \\
\hline
\end{tabular}

FDR, false discovery rate.

such as 'regulation of lymphocyte activation' and 'T-cell activation' (Fig. 5A). There was also enrichment for DEGs associated with cell components (CCs) including 'external side of the plasma membrane' and 'plasma membrane receptor complex' (Fig. 5B). Molecular function (MF) terms were markedly enriched in 'cytokine receptor binding and cytokine receptor activity' (Fig. 5C). In addition, compared with the $\mathrm{K}$ group, BPs in the I group included 'T-cell activation' and 'response to chemokine' (Fig. 6A). This group was also enriched in DEGs associated with the 'external side of the plasma membrane' and 'early endosome' CCs (Fig. 6B). MFs were markedly enriched in 'cytokine receptor binding' and 'receptor ligand activity' (Fig. 6C). Several BP enrichments were also observed in the $\mathrm{N}$ compared with the $\mathrm{K}$ group, including 'regulation of lymphocyte activation' and 'leukocyte cell-cell adhesion' (Fig. S3A). CCs were also markedly enriched in 'secretory granule membrane' and 'late endosome' (Fig. S3B). There was no MF enrichment in the $\mathrm{N}$ compared with the $\mathrm{K}$ group.
Table VI. Differentially expressed genes between group N and group $\mathrm{K}$.

\begin{tabular}{lclc}
\hline Gene symbol & Fold change & P-value & FDR P-value \\
\hline CD276 & 4.11 & 0.0347 & 0.5422 \\
IL6 & 4.54 & 0.0497 & 0.5422 \\
S100A8 & 9.49 & 0.0333 & 0.5422 \\
FCGR2B & 3.29 & 0.0363 & 0.5422 \\
TWIST1 & 2.65 & 0.004 & 0.5422 \\
CD68 & 2.25 & 0.0144 & 0.5422 \\
TYROBP & 3.22 & 0.0375 & 0.5422 \\
SNAI2 & 3.68 & 0.0212 & 0.5422 \\
MMP2 & 4.5 & 0.05 & 0.5422 \\
TGFB1 & 4.47 & 0.0019 & 0.5422 \\
\hline
\end{tabular}

FDR, false discovery rate.

KEGG pathway analysis between groups I and $\mathrm{N}$ revealed that all DEGs were primarily enriched in 'cytokine-cytokine receptor interaction' and 'viral protein interaction with cytokine and cytokine receptor' (Fig. 7A). A similar result was obtained for the I and $\mathrm{K}$ groups (Fig. 7B). KEGG pathway analysis in the $\mathrm{N}$ and $\mathrm{K}$ groups indicated that all the DEGs were primarily enriched in 'rheumatoid arthritis' and 'intestinal immune network for IgA production' (Fig. 7C). In general, these enrichments were associated with immune activation, suggesting that changes in gene expression during the inflammatory phase may affect the course and severity of disease. The functional annotations obtained based on different algorithms are roughly the same, because GO and predictions of KEGG are based on screened genes.

Experimental validation of hub gene expression. The expression of hub genes in the different groups was validated using RT-qPCR. According to bioinformatics analysis, primers were designed for the amplification of the top 10 hub genes in each group (Fig. 4D-F). Hub genes with $\mathrm{Cq}$ values ranging from 15-28 were considered highly expressed. $\mathrm{Cq}$ values $>28$ were considered to indicate low expression of the hub gene. The primers designed for the hub genes in the I and the $\mathrm{N}$ groups were specific for CCR1, CCR2, SELL, IL-10, CCR7, CD40LG, CD69, CXCL8, IL- 6 and CXCL9. The genes with a Cq value $<28$ included CCR1, CCR7, CD40LG, CXCL9, IL-6 and IL-10 (Fig. 8). The primers designed for the hub genes in the I and the $\mathrm{K}$ groups were specific for IL-10, ITGAM, ITGAX, IL-2, IL-4, IL-6, IL-13, IL-17A, FOXP3 and CD86. The genes with Cq values ranging from 15-28 included IL-10, IL-6, IL-13 and CD86 (Figs. 8 and 9; Table VII). The hub genes analyzed in groups N and K included CD276, IL-6, S100A8, FCGR2B, TWIST1, CD68, TYROBP, MMP2, SNAI2 and TGFB1. The genes with $\mathrm{Cq}$ values ranging from 15-28 included CD68 and MMP2 (Fig. 9).

Among the identified genes, IL-6 expression levels were significantly upregulated in the I group compared with the $\mathrm{N}$ group $(\mathrm{P}=0.0111)$. IL-6 expression levels were significantly 
A

GO_BP enrichment analysis of all DEGs
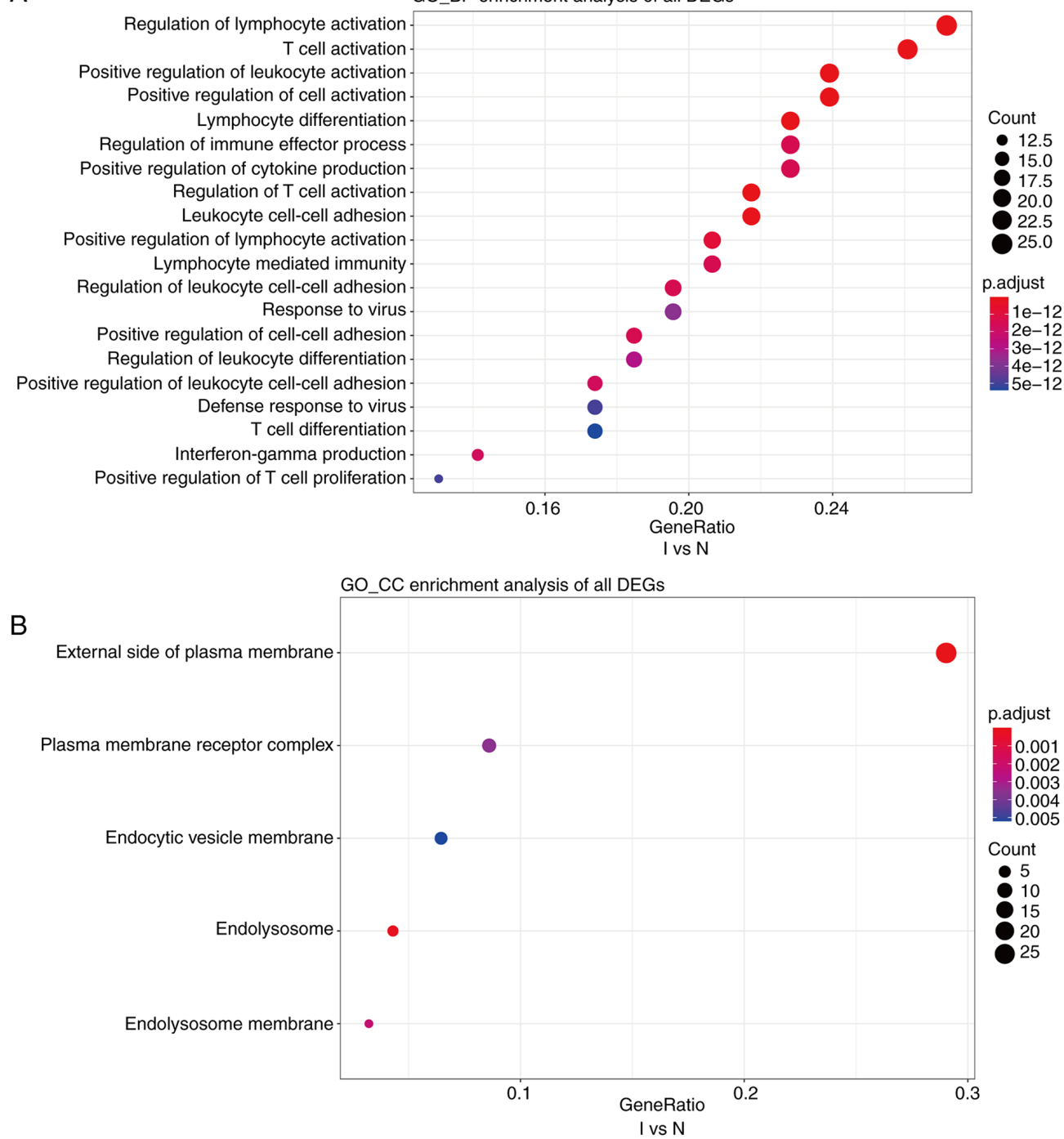

C

GO_MF enrichment analysis of all DEGs

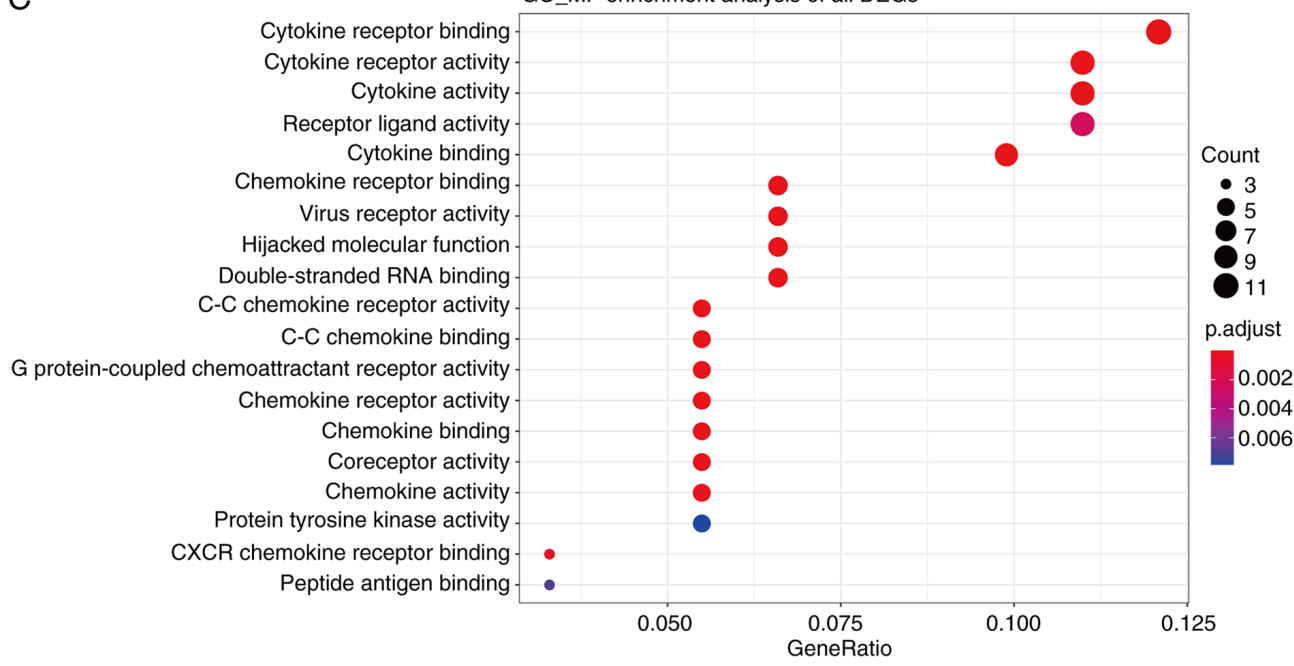

I vs N

Figure 5. GO, a database created by the Gene Ontology Consortium, consists of a set of pre-defined GO terms that define and describe the functions of genes and proteins. (A-C) GO enrichment analyses of biological processes, cellular components, and molecular functions of differentially expressed genes between groups I and $\mathrm{N}$. The cutoff for $\log 2$ fold change $>1.5$ or $<-0.5$ and $\mathrm{P}<0.05$ were used as screening criteria. GO, Gene Ontology; BP, biological processes; $\mathrm{CC}$, cellular components; MF, molecular functions.

upregulated in the $\mathrm{K}$ group compared with the $\mathrm{N}(\mathrm{P}=0.0112)$. Compared with that in healthy skin tissue, CCR7 expression was upregulated in inflamed tissue and downregulated in keloid tissue (Table VIII; Fig. 8). Western blot analysis revealed 
A
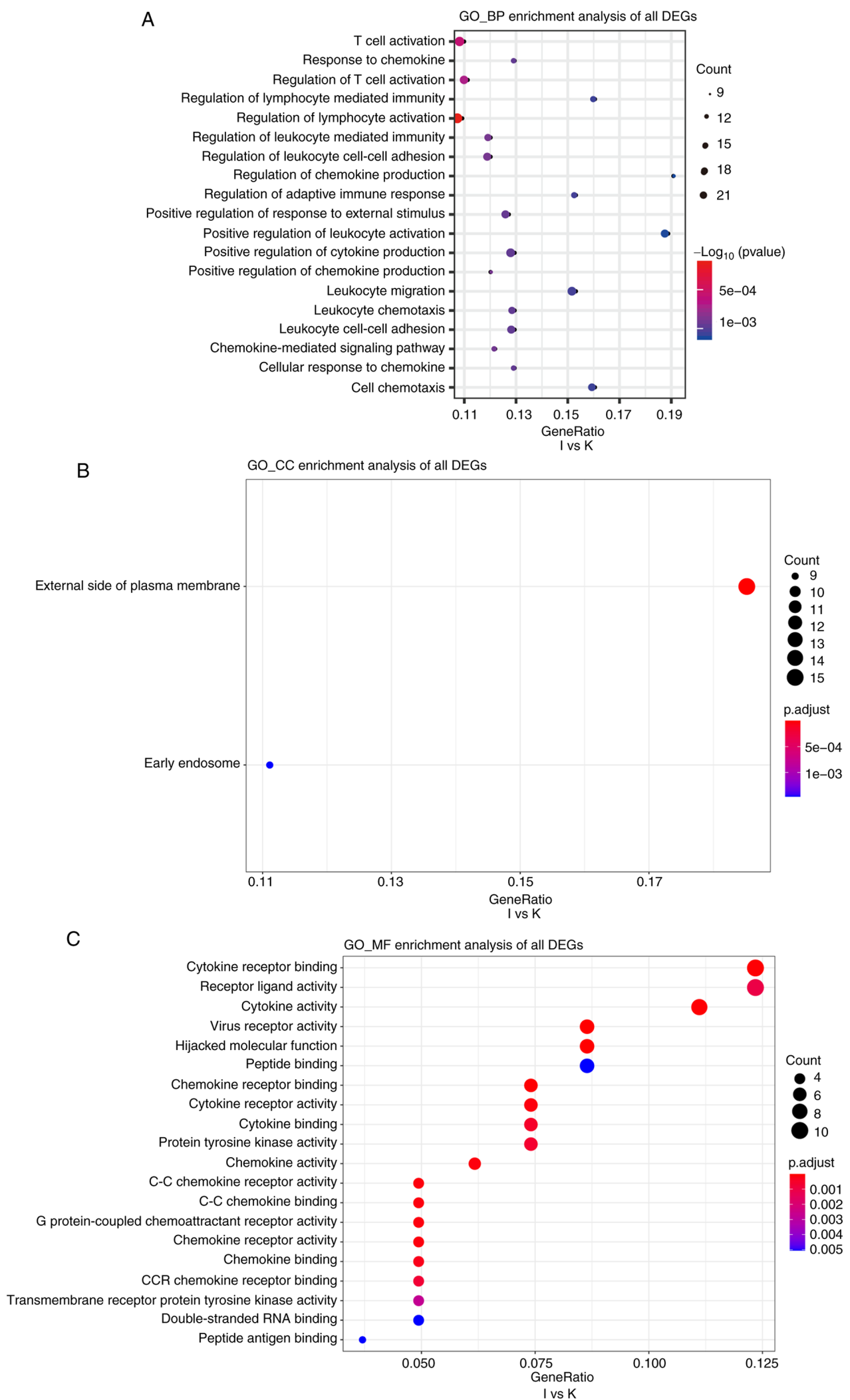

Figure 6. (A-C) Gene Ontology enrichment analyses of biological processes, cellular components, and molecular functions of differentially expressed genes between groups I and $\mathrm{K}$. The cutoff for $\log 2$ fold change $>1.5$ or $<-0.5$ and $\mathrm{P}<0.05$ were used as screening criteria. GO, Gene Ontology; BP, biological processes; $\mathrm{CC}$, cellular components; MF, molecular functions.

that the relative expression levels of CCR7 were significantly different in the normal, inflamed and keloid tissue $(\mathrm{P}<0.017$; Fig. 9D and E), indicating that CCR7 may be important in keloid pathogenesis.

\section{Discussion}

Tumors are regulated by their local immune microenvironment (21-24), and keloids share certain characteristics with 
A

$$
\begin{array}{r}
\text { Cytokine-cytokine receptor interaction } \\
\text { Viral protein interaction with cytokine and cytokine receptor } \\
\text { Malaria } \\
\text { Cell adhesion molecules (CAMs) } \\
\text { Allograft rejection } \\
\text { Autoimmune thyroid disease } \\
\text { Chemokine signaling pathway } \\
\text { Kaposi sarcoma-associated herpesvirus infection } \\
\text { Graft-versus-host disease } \\
\text { JAK-STAT signaling pathway } \\
\text { Influenza A } \\
\text { Measles } \\
\text { Toll-like receptor signaling pathway } \\
\text { Intestinal immune network for IgA production } \\
\text { Pertussis } \\
\text { Viral myocarditis } \\
\text { Rheumatoid arthritis } \\
\text { Type I diabetes mellitus } \\
\text { Th17 cell differentiation } \\
\text { Natural killer cell mediated cytotoxicity }
\end{array}
$$

B
KEGG enrichment analysis of all DEGs

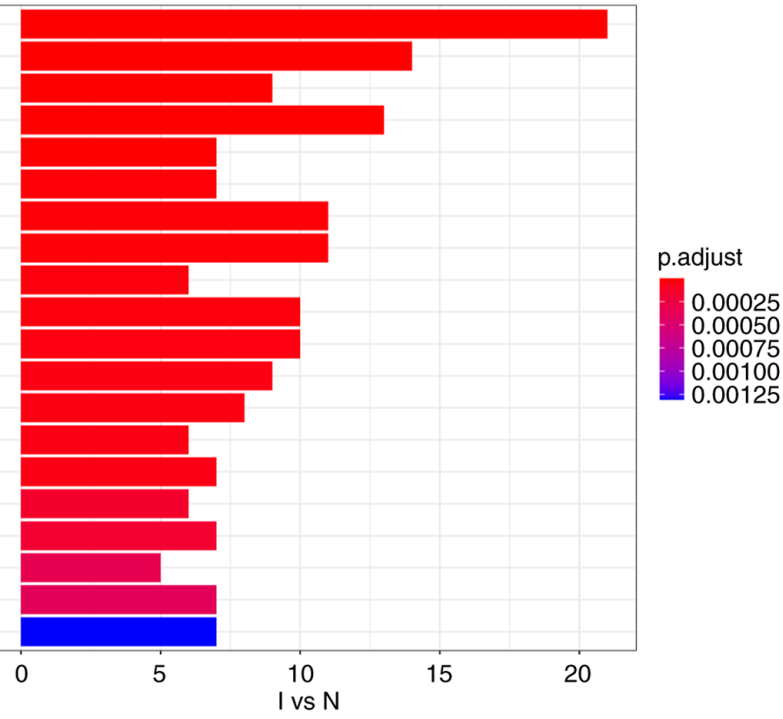

KEGG enrichment analysis of all DEGs

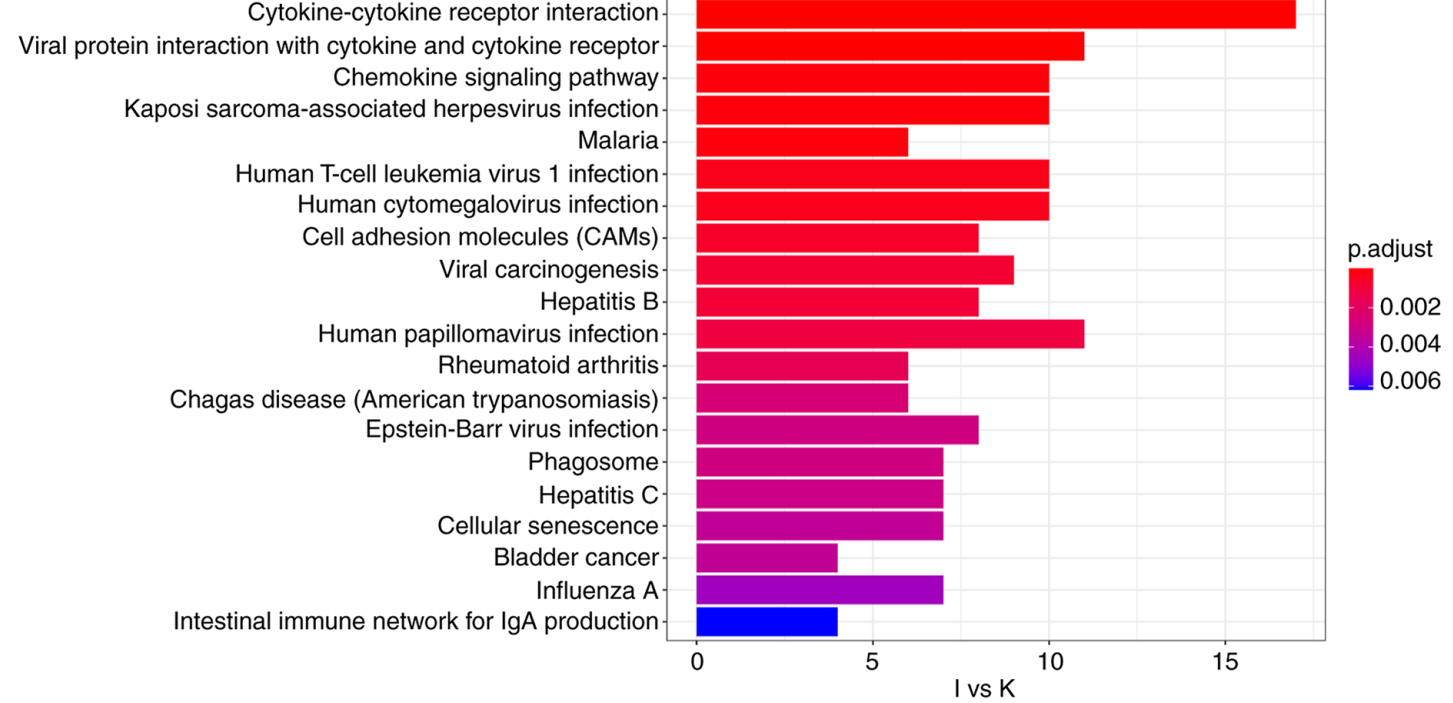

C

KEGG enrichment analysis of all DEGs

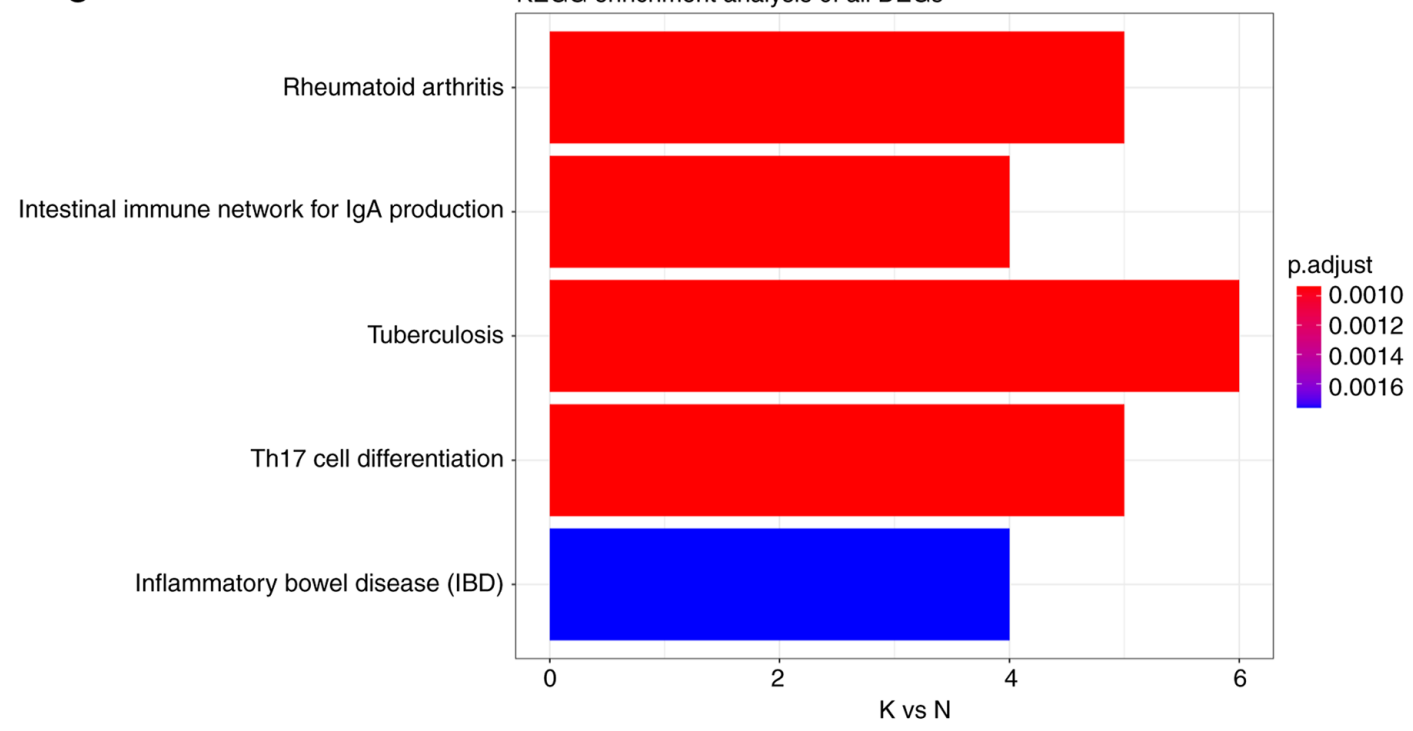

Figure 7. (A) KEGG pathway analysis of DEGs between groups I and N. The cutoff for $\log 2$ fold change $>1.5$ or $<-0.5$ and $\mathrm{P}<0.05$ were used as screening criteria. (B) KEGG pathway analysis of DEGs between groups I and $\mathrm{K}$. The cutoff for log 2 fold change $>1.5$ or $<-0.5$ and $\mathrm{P}<0.05$ were used as screening criteria. (C) KEGG pathway analysis of DEGs between groups N and K. The cutoff for $\log 2$ fold change $>1.5$ or $<-0.5$ and P $<0.05$ were used as screening criteria. KEGG, Kyoto Encyclopedia of Genes and Genomes; DEGs, differentially expressed genes. 
A

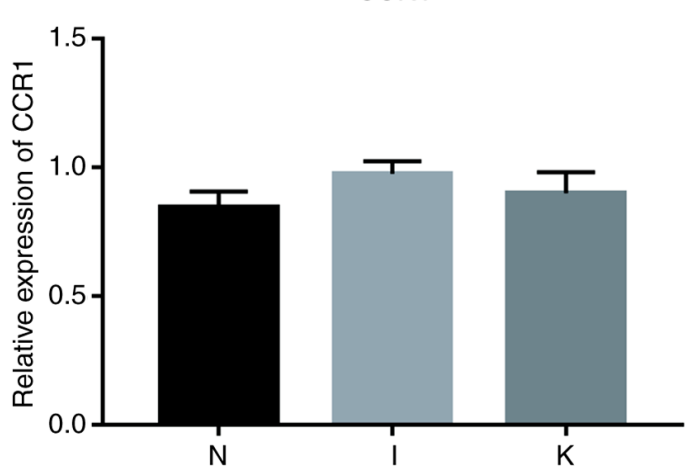

C

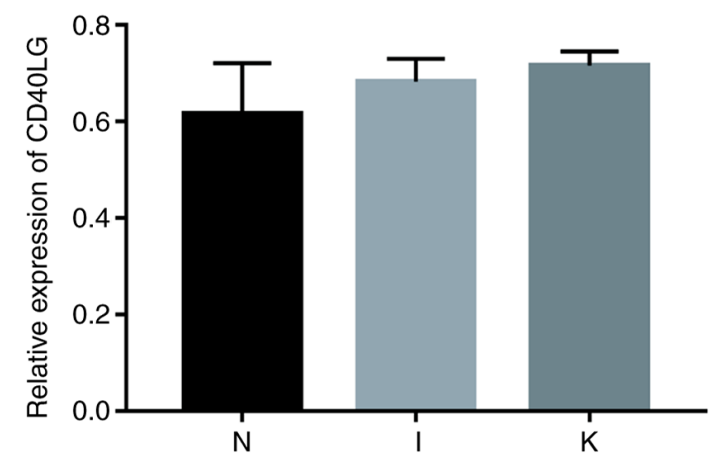

E

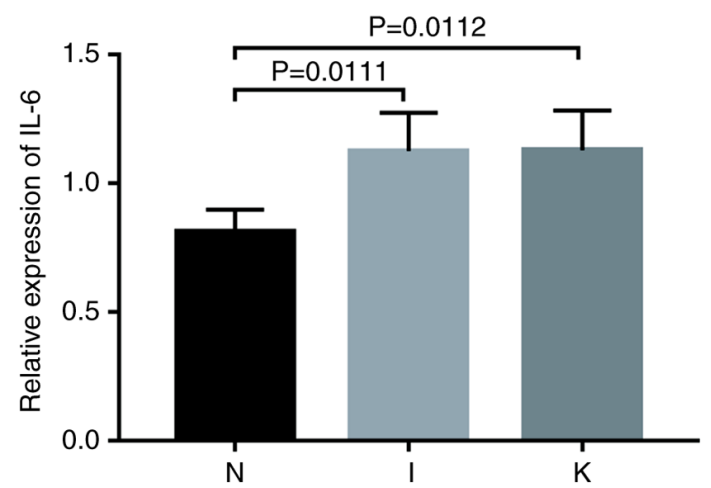

B

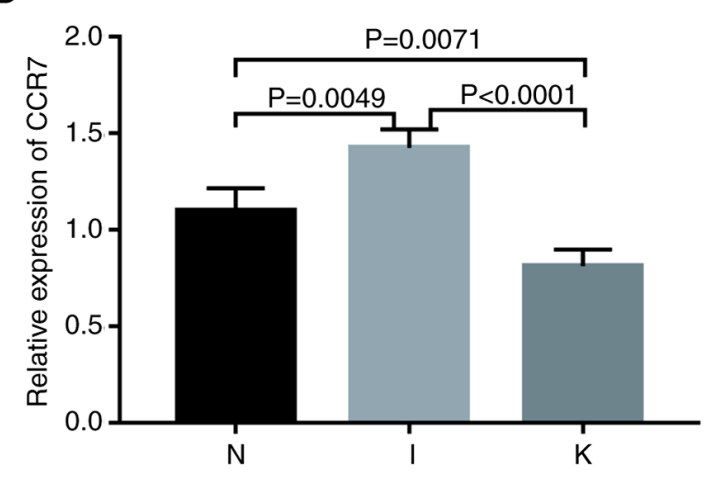

D

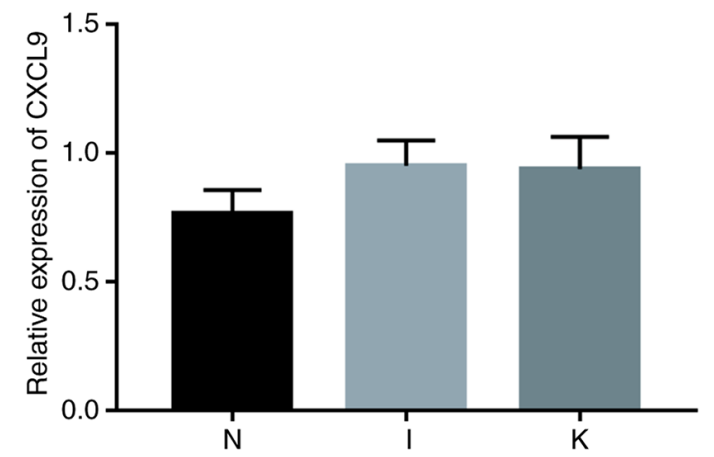

$\mathrm{F}$

IL-10

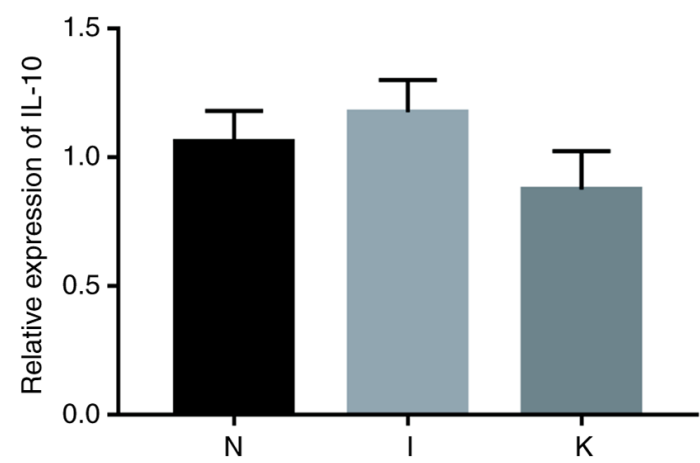

Figure 8. Relative expression of genes. $\mathrm{P}<0.017$ was considered to indicate a statistically significant difference. (A) Relative expression of CCR1 by RT-qPCR analysis. (B) Relative expression of CCR7 by RT-qPCR analysis. (C) Relative expression of CD40LG by RT-qPCR analysis. (D) Relative expression of CXCL9 by RT-qPCR analysis. (E) Relative expression of IL-6 by RT-qPCR analysis. (F) Relative expression of IL-10 by RT-qPCR analysis. RT-qPCR, reverse transcription-quantitative PCR.

tumors. Abnormal expression of immune-related genes likely underlies the formation of keloids. Aberrant gene expression often originates from congenital risks, although external stimuli, such as inflammation, may also be a trigger.

In the present study, to examine the pathogenesis of keloids, healthy skin, inflamed and keloid tissue were collected from patients with keloids and analyzed. Hub genes were identified in the $\mathrm{N}$ group compared with the I group, including CCR1, CCR7, CD40LG, CXCL9, IL-6 and IL-10. Pro-inflammatory effects have previously been demonstrated in animal models of CCR1 neurological disease. For instance, Yan et al (17) demonstrated that CCR1 activation could promote an intracerebral inflammatory response in mice via the CCR1/topless-related protein
1/ERK1/2 signaling pathway. CCR1 may play a pro-inflammatory role in the formation of keloids. CD40LG is expressed on the surface of $\mathrm{T}$ cells and regulates B-cell function by activating CD40 on the surface of B cells. Non-hematopoietic cells expressing CD40 can also activate CD40LG and trigger a pro-inflammatory response (25). CD40LG may also play a pro-inflammatory role in the formation of keloids. CXCL9 binds to CXCR3, affecting the proliferation of cells involved in immune and inflammatory responses and chemotaxis of activated $\mathrm{T}$ cells. The CXCL9, -10 and $-11 / \mathrm{CXCR} 3$ axis regulates the migration, differentiation and activation of immune cells, such as cytotoxic T lymphocytes, natural killer cells and macrophages. In addition, T helper (Th) 1 polarization via this 
A

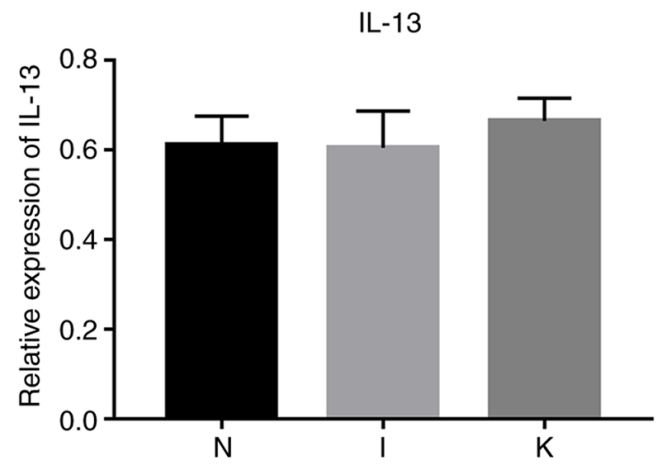

C

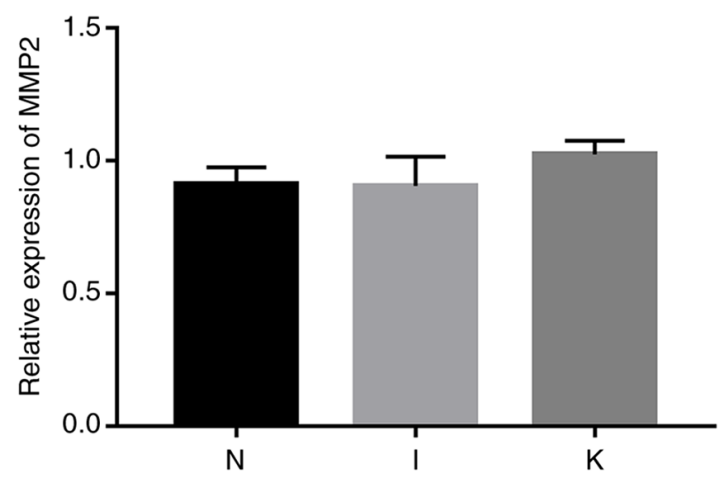

$\mathrm{B}$

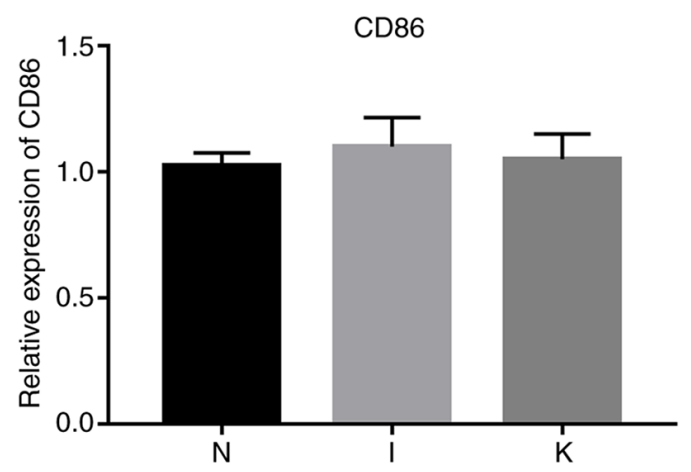

D

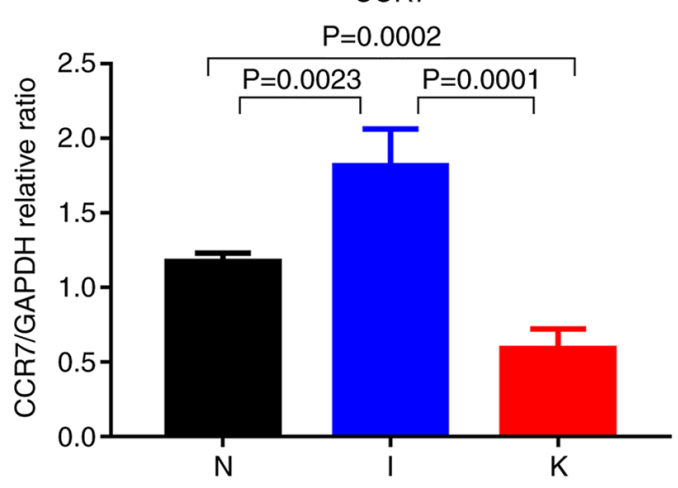

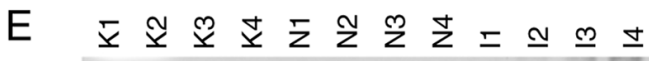

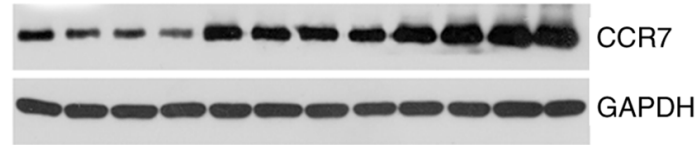

Figure 9. (A-C) Relative expression of CD86, and MMP2 by reverse transcription-quantitative PCR analysis. (D) Quantitative comparison of CCR7 expression among the three groups. (E) Western blot expression of CCR7 among the three groups ( $\mathrm{N}$, I and $\mathrm{K}$ group). P<0.0001, I vs. N; $\mathrm{P}=0.0023$, I vs. K; $\mathrm{P}=0.0001$, $\mathrm{N}$ vs. $\mathrm{K}$; and $\mathrm{P}=0.0002$. $\mathrm{P}<0.017$ was considered to indicate a statistically significant difference.

axis also activates immune responses to IFN- $\gamma(26)$. These hub genes may be important in the early stages of keloid formation, from healthy skin to the inflammation stage, although further research is required to elucidate the role of each of these hub genes.

The hub genes identified in the I group compared with the $\mathrm{K}$ group included IL-10, IL-6, IL-13 and CD86. IL-10 has been reported to significantly inhibit the proliferation of keloid fibroblasts $(6,8,27)$. IL-10 expression was the highest in inflamed tissue samples and lowest in the K group. The expression levels of IL-10 was firstly increased, then decreased in the three groups. It may be hypothesized that the occurrence and development of keloids may be associated with changes in IL-10 expression, although the underlying mechanism requires further study. High expression of IL-10 in inflamed tissue may inhibit inflammatory damage in keloids and slow down the progression of the disease. IL-13 and CD86 did not differ significantly between the three groups, although there was a trend towards increasing expression from healthy skin, to inflamed and finally to keloid samples. Zhang et al (21) suggested that the expression levels of IL- 6 and IL-17 in keloids were significantly increased. Keloid lesions also exhibit enhanced IL-4/IL-13 signaling and a Th2-dominated immune response (7). In the present study, the expression levels of IL- 6 were the lowest in the $\mathrm{N}$ group and significantly increased in the I group compared with the $\mathrm{N}$ group. There was no significant change in the I and the K groups. IL-6 is a key pro-inflammatory cytokine. Upregulation of IL-6 in keloid fibroblasts leads to an increase in downstream target gene expression, including in genes associated with cell proliferation and matrix synthesis (28). IL-6 upregulation in keloids may lead to an intense inflammatory response and subsequent production of more collagen fibers. The frequency of Foxp $3^{+}$regulatory $\mathrm{T}$ cells (Tregs) in keloid tissue is significantly higher than that in peripheral blood. In addition, macrophages from keloid tissue have a strong ability to induce Foxp3 expression in circulating $\mathrm{CD}^{+} \mathrm{T}$-cells and may promote Treg differentiation by upregulating Foxp3 expression (29). CD86 is a T-lymphocyte activation antigen that participates in T-lymphocyte proliferation and IL-2 production by binding to CD28 or CTLA-4 (30). All these hub genes have different functions in the initiation and regulation of immune responses. They may be important in the later stages of keloid formation, from the inflammatory stage to the keloid formation stage.

The roles played by several genes in keloid development need to be explored further, including CCR1, CCR7, CD40LG, 
Table VII. Comparison of the median level of hub genes among various groups (N, I and K).

\begin{tabular}{llccr}
\hline & \multicolumn{3}{c}{ Mean value, IQR for: } \\
\cline { 2 - 4 } Hub genes & \multicolumn{1}{c}{ Group N } & \multicolumn{1}{c}{ Group I } & Group K & P-value \\
\hline CCR1 & $0.85,0.8-0.91$ & $0.97,0.90-1.00$ & $0.90,0.82-0.97$ & 0.0600 \\
CCR7 & $1.10,1-1.2$ & $1.42,1.32-1.5$ & $0.81,0.72-0.88$ & $<0.0001$ \\
CD40LG & $0.63,0.53-0.70$ & $0.68,0.65-0.72$ & $0.71,0.69-0.74$ & 0.3030 \\
CXCL9 & $0.76,0.70-0.86$ & $0.95,0.85-1.00$ & $0.93,0.81-1.00$ & 0.0632 \\
IL-6 & $0.81,0.73-0.89$ & $1.13,1.00-1.27$ & $1.13,1.11-1.28$ & 0.0124 \\
IL-10 & $1.06,1.00-1.18$ & $1.18,1.05-1.28$ & $0.88,0.73-1.00$ & 0.0314 \\
IL-13 & $0.61,0.56-0.67$ & $0.60,0.53-0.68$ & $0.67,0.61-0.71$ & 0.4172 \\
CD86 & $1.03,1.00-1.08$ & $1.10,1.00-1.20$ & $1.05,1.00-1.2$ & 0.5320 \\
MMP2 & $0.91,0.86-0.98$ & $0.91,0.80-1.00$ & $1.03,1.00-1.08$ & 0.1072 \\
\hline
\end{tabular}

Table VIII. Promising biomarkers of keloid via reverse transcription-quantitative PCR.

\begin{tabular}{lcccc}
\hline & & \multicolumn{3}{c}{ Bonferroni's correction $^{\mathrm{a}}$} \\
\cline { 3 - 5 } Gene & Anova & N vs. I & I vs. K & N vs. K \\
\hline CCR7 & $\mathrm{P}<0.0001$ & 0.0049 & $<0.0001$ & 0.0071 \\
IL-6 & $\mathrm{P}=0.0124$ & 0.0111 & $\mathrm{NS}$ & 0.0112 \\
IL-10 & $\mathrm{P}=0.0314$ & $\mathrm{NS}$ & $\mathrm{NS}$ & $\mathrm{NS}$ \\
\hline
\end{tabular}

${ }^{a}$ The P-value was considered statistically significant at a Bonferroni corrected P-value below 0.017. NS, not significant.

CXCL9, IL-6, IL-10, IL-13, CD86 and MMP2. Inflammation is an important mechanism in the pathogenesis of keloids. CCR7 is a receptor protein with a seven-pass transmembrane structure conjugated with allotrope G protein, which is expressed in a variety of lymphoid tissue types (31). Under normal physiological conditions, CCR7 could activate both B- and T-lymphocytes, participate in the homing of T-lymphocytes, affect the transport of T-lymphocytes in lymph nodes and stimulate the maturation of dendritic cells (31). During the inflammatory process, abnormal expression of CCR7 may occur. The binding of chemokines to their receptors induces leukocytes to move towards sites of inflammation. CCR7 is important in the process of tumorigenesis and development by promoting the invasion and migration of tumor cells. In response to inflammatory stimulation, the expression of CCR7 increases to recruit more $\mathrm{B}$ - and T-cells to participate in the immune response. In the present study, expression of CCR7 tended to increase in the I group. In the late stage of keloid formation, the expression of CCR7 in the K group decreased to a lower level compared with the other two groups. CCR7 expression was significantly different between the I and $\mathrm{K}$ groups, which represents a transition of the patient from an inflamed to a keloid state $(\mathrm{P}<0.0001)$. This difference was more significant at the protein level, with the highest CCR7 protein expression levels being in the I group and the lowest in the K group. This suggested that CCR7 may play an important role during the formation of keloids. CCR7 may be a promising biomarker for the treatment of keloid, although the specific mechanism of action still needs to be further studied.

In the present study, BP terms were identified between groups I and $\mathrm{N}$, including 'regulation of lymphocyte activation' and 'T-cell activation'. A similar result was observed between groups I and K. A study conducted by Huang et al (32) suggested that upregulated mRNA transcripts were involved in cell proliferation and tissue repair, whereas downregulated transcripts were involved in apoptosis.

In the present study, KEGG pathway analysis between groups I and $\mathrm{N}$ revealed that the identified DEGs were primarily enriched in 'cytokine-cytokine receptor interactions' and 'viral protein interactions with cytokines and cytokine receptors', indicating that these pathways may affect keloid formation. A study performed by Zhong et al (27) identified target genes that were associated with the MAPK and the hypoxia-inducible factor-1 signaling pathway. Immune regulation is crucial to the immune response of the body, and cytokines are important in this process. In the face of injury, inflammation or tumors, impaired activation of the immune system through cytokines and their receptors could dampen the immune response (33). Conversely, excessively strong immune responses may trigger autoimmune diseases. There are several important immune cytokines, such as IL-2, -3, -4, -5, -6, -9, -10, -12, -13 and -14, as well as IFN- $\gamma(24,33)$. In the present study, IL-6, IL-10 and IL-13 were identified as playing an important role in patients with keloids. In future, functional studies on these promising biomarkers may be conducted via immune absorbent spot (ELISpot). In addition, in future studies, cell functions of these promising biomarkers may be studied. Furthermore, the "cytokine-cytokine receptor interactions' pathway was identified in the current KEGG analysis. These results suggested that the pathogenesis of keloids may be associated with 'cytokine-cytokine receptor interactions'. Future research into the molecular mechanisms of keloid pathogenesis should explore these possibilities. KEGG analysis suggested that the pathogenesis of keloids was associated with 'viral protein interactions with cytokines and cytokine receptors'. These results suggested that the pathogenesis of keloids may be related to viral infections. Future research into the molecular mechanisms of keloid pathogenesis should explore these possibilities. 
Despite the rigorous bioinformatics analysis involved in the present study, there are some limitations. It was difficult to obtain inflammatory tissue, which may have led to some deviation of results. The remaining sample size was too small to continue to use for PCR after sequencing, especially the inflammatory tissue content, and considering, the result is more general using other samples to perform the verification. The sample size in the present study was small and should be increased in future studies. Additionally, the present study lacks in-depth functional experiments in cell and animal models.

In conclusion, immune-related DEGs were identified in healthy skin, inflamed and keloid tissue samples from patients with keloids. Certain of these hub genes may be key in the formation of keloids, such as CCR7, IL-6 and IL-10 (Table VIII). These may represent important targets for drug therapy and precise treatment of keloids. Regulating these key genes may help prevent, alleviate or even cure keloids. These results also provided further insight into keloid pathogenesis.

\section{Acknowledgements}

Not applicable.

\section{Funding}

The present study was supported by The National Natural Science Foundation of China (grant no. 81871538).

\section{Availability of data and materials}

The datasets used and/or analyzed during the current study are available from the corresponding author on reasonable request. The data has been uploaded to the NCBI website (SUB10443300).

\section{Authors' contributions}

MS performed the experiments, carried out the data analysis and was a major contributor to preparation of the manuscript. HL, KS, SL and YH collected samples and performed the experiments. YW made substantial contributions to the design of the study. KS, SL and YH were major contributors to the submission of the manuscript and provided technical support in the experimental methods. MS, HL and YW confirm the authenticity of all the raw data. All authors read and approved the final manuscript.

\section{Ethics approval and consent to participate}

The experimental plan for the present study was approved (approval no. JS-2907) by The Medical Ethics Committee of Peking Union Medical College Hospital (Beijing, China). Written and photographic informed consents were obtained from all participants.

\section{Patient consent for publication}

All patients or guardians of the patients provided written informed consent for the publication of any associated data and accompanying images.

\section{Competing interests}

The authors declare that they have no competing interests.

\section{References}

1. Brown JJ, Ollier W, Arscott G, Ke X, Lamb J, Day P and Bayat A: Genetic susceptibility to keloid scarring: SMAD gene SNP frequencies in Afro-caribbeans. Exp Dermatol 17: 610-613, 2008.

2. Chung S, Nakashima M, Zembutsu $\mathrm{H}$ and Nakamura Y: Possible involvement of NEDD4 in keloid formation; its critical role in fibroblast proliferation and collagen production. Proc Jpn Acad Ser B Phys Biol Sci 87: 563-573, 2011.

3. Glass DA II: Current understanding of the genetic causes of keloid formation. J Investig Dermatol Symp Proc 18: S50-S53, 2017.

4. Tsai $\mathrm{CH}$ and Ogawa R: Keloid research: Current status and future directions. Scars Burn Heal 5: 2059513119868659, 2019.

5. Song KX, Liu S, Zhang MZ, Liang WZ, Liu H, Dong XH, Wang YB and Wang XJ: Hyperbaric oxygen therapy improves the effect of keloid surgery and radiotherapy by reducing the recurrence rate. J Zhejiang Univ Sci B 19: 853-862, 2018.

6. Ogawa R: Keloid and hypertrophic scars are the result of chronic inflammation in the reticular dermis. Int J Mol Sci 18: 606, 2017.

7. Diaz A, Tan K, He H, Xu H, Cueto I, Pavel AB, Krueger JG and Guttman-Yassky E: Keloid lesions show increased IL-4/IL-13 signaling and respond to Th2-targeting dupilumab therapy. J Eur Acad Dermatol Venereol 34: e161-e164, 2020.

8. Shi CK, Zhao YP, Ge P and Huang GB: Therapeutic effect of interleukin-10 in keloid fibroblasts by suppression of TGF- $\beta / \mathrm{Smad}$ pathway. Eur Rev Med Pharmacol Sci 23: 9085-9092, 2019.

9. Gankande TU, Wood FM, Edgar DW, Duke JM, DeJong HM, Henderson AE and Wallace HJ: A modified vancouver scar scale linked with TBSA (mVSS-TBSA): Inter-rater reliability of an innovative burn scar assessment method. Burns 39: 1142-1149, 2013.

10. Wang CH, Shan MJ, Liu H, Hao Y, Song KX, Wu HW, Meng T, Feng C, Qi Z, Wang Z and Wang YB: Hyperbaric oxygen treatment on keloid tumor immune gene expression. Chin Med J (Engl) 134: 2205-2213, 2021.

11. Privé F, Luu K, Vilhjálmsson BJ and Blum M: Performing highly efficient genome scans for local adaptation with $\mathrm{r}$ package pcadapt version 4. Mol Biol Evol 37: 2153-2154, 2020.

12. Kanehisa M and Goto S: KEGG: Kyoto encyclopedia of genes and genomes. Nucleic Acids Res 28: 27-30, 2000.

13. Huang DW, Sherman BT and Lempicki RA: Systematic and integrative analysis of large gene lists using DAVID bioinformatics resources. Nat Protoc 4: 44-57, 2009.

14. Szklarczyk D, Gable AL, Lyon D, Junge A, Wyder S, Huerta-Cepas J, Simonovic M, Doncheva NT, Morris JH and Bork P: STRING v11: Protein-protein association networks with increased coverage, supporting functional discovery in genome-wide experimental datasets. Nucleic Acids Res 47: D607-D613, 2019.

15. Shannon P, Markiel A, Ozier O, Baliga NS, Wang JT, Ramage D, Amin N, Schwikowski B and Ideker T: Cytoscape: A software environment for integrated models of biomolecular interaction networks. Genome Res 13: 2498-2504, 2003.

16. Ni M, Liu X, Wu J, Zhang D, Tian J, Wang T, Liu S, Meng Z, Wang K, Duan X, et al: Identification of candidate biomarkers correlated with the pathogenesis and prognosis of non-small cell lung cancer via integrated bioinformatics analysis. Front Genet 9: 469, 2018.

17. Yan J, Zuo G, Sherchan P, Huang L, Ocak U, Xu W Travis Z, Wang W, Zhang J and Tang J: CCR1 Activation Promotes Neuroinflammation Through CCR1/TPR1/ERK1/2 Signaling Pathway After Intracerebral Hemorrhage in Mice. Neurotherapeutics 17: 1170-1183, 2020.

18. Chin CH, Chen SH, Wu HH, Ho CW, Ko MT and Lin CY: cytoHubba: Identifying hub objects and sub-networks from complex interactome. BMC Syst Biol 8 (Suppl 4): S11, 2014.

19. Song X, Du R, Gui H, Zhou M, Zhong W, Mao C and Ma J: Identification of potential hub genes related to the progression and prognosis of hepatocellular carcinoma through integrated bioinformatics analysis. Oncol Rep 43: 133-146, 2020.

20. Livak KJ and Schmittgen TD: Analysis of relative gene expression data using real-time quantitative PCR and the 2-(Delta Delta C(T)) method. Methods 25: 402-408, 2001. 
21. Zhang Q, Yamaza T, Kelly AP, Shi S, Wang S, Brown J, Wang L, French SW, Shi S and Le AD: Tumor-like stem cells derived from human keloid are governed by the inflammatory niche driven by IL-17/IL-6 axis. PLoS One 4: e7798, 2009.

22. Limandjaja GC, Niessen FB, Scheper RJ and Gibbs S: The keloid disorder: Heterogeneity, histopathology, mechanisms and models. Front Cell Dev Biol 8: 360, 2020.

23. van den Broek LJ, Limandjaja GC, Niessen FB and Gibbs S: Human hypertrophic and keloid scar models: Principles, limitations and future challenges from a tissue engineering perspective. Exp Dermatol 23: 382-386, 2014.

24. Tan S, Khumalo $\mathrm{N}$ and Bayat A: Understanding keloid pathobiology from a quasi-neoplastic perspective: Less of a sca and more of a chronic inflammatory disease with cancer-like tendencies. Front Immunol 10: 1810, 2019.

25. Karnell JL, Rieder SA, Ettinger R and Kolbeck R: Targeting the CD40-CD40L pathway in autoimmune diseases: Humora immunity and beyond. Adv Drug Deliv Rev 141: 92-103, 2019.

26. Tokunaga R, Zhang W, Naseem M, Puccini A, Berger MD, Soni S, McSkane M, Baba H and Lenz HJ: CXCL9, CXCL10, CXCL11/CXCR3 axis for immune activation-A target for novel cancer therapy. Cancer Treat Rev 63: 40-47, 2018.

27. Zhong L, Bian L, Lyu J, Jin H, Liu Z, Lyu L and Lu D: Identification and integrated analysis of microRNA expression profiles in keloid. J Cosmet Dermatol 17: 917-924, 2018
28. Ghazizadeh M: Essential role of IL-6 signaling pathway in keloid pathogenesis. J Nippon Med Sch 74: 11-22, 2007.

29. Jin Q, Gui L, Niu F, Yu B, Lauda N, Liu J, Mao X and Chen Y: Macrophages in keloid are potent at promoting the differentiation and function of regulatory T cells. Exp Cell Res 362: 472-476, 2018.

30. Suzuki M, Yokota M, Matsumoto T and Ozaki S: Synergic effects of CD40 and CD86 silencing in dendritic cells on the control of allergic diseases. Int Arch Allergy Immunol 177: 87-96, 2018.

31. Mburu YK, Wang J, Wood MA, Walker WH and Ferris RL: CCR7 mediates inflammation-associated tumor progression. Immunol Res 36: 61-72, 2006.

32. Huang H, Fu S and Liu D: Detection and analysis of the hedgehog signaling pathway-related long non-coding RNA (lncRNA) expression profiles in keloid. Med Sci Monit 24: 9032-9044, 2018.

33. Spangler JB, Moraga I, Mendoza JL and Garcia KC: Insights into cytokine-receptor interactions from cytokine engineering. Annu Rev Immunol 33: 139-167, 2015.

This work is licensed under a Creative Commons Attribution-NonCommercial-NoDerivatives 4.0 International (CC BY-NC-ND 4.0) License. 